\title{
Long Interfascicular Axon Growth from Embryonic Neurons Transplanted into Adult Myelinated Tracts
}

\author{
Stephen J. A. Davies, Pauline M. Field, and Geoffrey Raisman \\ Norman and Sadie Lee Research Centre, Laboratory of Neurobiology, National Institute for Medical Research, London \\ NW7 1AA, United Kingdom
}

In a previous study we used the species-specific marker M6 to demonstrate that transplanted mouse embryonic hippocampal neurons grow axons at a rate of at least $1 \mathrm{~mm} / \mathrm{d}$ for a distance of at least $10 \mathrm{~mm}$ along the longitudinal axis of the fimbria in immunosuppressed adult rat hosts. We now show that hippocampal neurons are able to grow comparably long interfascicular axons in two other myelinated adult fiber tracts, the corpus callosum and the cingulum. Moreover, suspensions of cells from embryonic neocortex and superior colliculus transplanted into each of these three adult host sites also give interfascicular axon growth whose speed, intensity, and pattern of distribution are identical to those of transplanted hippocampal neurons.

The axons of the donor cells grow in both directions along the longitudinal axis of the host tracts, where they are interspersed in parallel among the normal host axons, the rows of host interfascicular glial nuclei, and the longitudinal processes of host tract astrocytes. Serial section analysis through the complex trajectories of the host fiber bundles of the fimbria and corpus callosum shows that the course of the donor axons conforms to the underlying orientation of the axonal and glial structures of the host fiber tract.

These observations indicate that long interfascicular axon growth can occur in several different adult myelinated fiber tracts. The donor axons become integrated with the host tract fibers and glia, and they respect intertract boundaries. Growth is not restricted to the types of axons normally present in the tracts.

IKey words: axon guidance, hippocampus, vimentin, glianeuron interactions, transplant, regeneration, myelinated tracts]

There is a generally held view that the failure of regeneration after brain and spinal cord injuries is due to the inability of axons to grow along adult ccntral fibcr tracts (Ramon y Cajal, 1928). It was therefore a surprise to find that axons of transplanted embryonic neurons can grow rapidly for considerable distances along the fiber tracts of adult brain and spinal cord (Tønder et al., 1990; Fujii, 1991; Wictorin et al., 1991, 1992; Strömberg et al., 1992).

Received May 7, 1993; revised Aug. 2, 1993; accepted Aug. 26, 1993.

We are grateful to Ursula Harris for expert assistance, to Dr. Carl Lagenaur and Professor Ray I und for the M6 antibody, and to the International Spinal Research Trust for vital financial assistance.

Correspondence should be addressed to S. J. A. Davies, Laboratory of Neurobiology, National Institute for Medical Research, The Ridgeway, Mill Hill, London NW7 IAA, UK.

Copyright (C) 1994 Society for Neuroscience 0270-6474/94/141596-17\$05.00/0
These observations raise a number of questions: Is the ability to sustain long interfascicular axon growth (LIAG) a general property of all adult fiber tracts? Is the growth in a fiber tract restricted to the types of axons normally present in that tract? And what is the tissue substrate for axon growth?

In a previous study using a microtransplantation technique (Emmett et al., 1990) that causes minimal disturbance to the arrangement of the host axons and glia, we used two mousespecific axonal markers, M6 and Thy-1.2, to demonstrate that late embryonic hippocampal donor neurons are able to grow axons at about $1-2 \mathrm{~mm} / \mathrm{d}$ along the adult fimbria (Davies et al., 1993). In the present study we have extended this approach to two other adult central tracts-the corpus callosum and the cingulum. By using cell suspensions from three different embryonic areas - the hippocampus, the neocortex, and the superior colliculus - we have explored the need for specificity of matching of donor cells and host pathways. Using a serial section analysis we have compared the orientation of the donor axons with the underlying axonal and glial arrangement of the host fiber tracts, and examined whether the course of the newly growing axons respects the boundaries between adjacent tracts

\section{Materials and Methods}

Donor material. Cell suspensions at a concentration of $8-20 \times 10^{6} \mathrm{cells} /$ $\mathrm{ml}$ were prepared from hippocampus, parietal neocortex, and superior colliculus dissected from F,14 and F18 CBA(II) mouse embryos (EO = day of vaginal plugs) and kept in a defined medium (Bottenstein and Sato, 1979) on ice.

Transplantation. A pulsed air pressure system (Emmett et al., 1990) was used to inject $0.5 \mu \mathrm{l}$ of suspension (containing $0.4-1.0 \times 10^{4}$ cells) through a glass micropipette $(50 \mu \mathrm{m}$ i.d., with a beveled tip) into one of three sites in the left hemisphere-the medial part of the fimbria, the rostrum of the corpus callosum, or the cingulum -in adult female ASstrain rats, body weight $180-200 \mathrm{gm}$, under tribromoethanol anesthesia ( $20 \mathrm{mg} / 100 \mathrm{gm}$ body weight, i.p.). The stereotaxic coordinates, measured from the bregma with the head held in the flat skull position, were fimbria: $1.6 \mathrm{~mm}$ caudal, $1.3 \mathrm{~mm}$ lateral, $4.1 \mathrm{~mm}$ ventral; corpus callosum: $1.4,1.2$, and $3.0 \mathrm{~mm}$; and cingulum: $1.4,0.9$, and $3.0 \mathrm{~mm}$. Graft rejection was suppressed by maintaining the animals from the time of operation on cyclosporin A (Sandimmun, Sandoz; $10 \mathrm{mg} / 100 \mathrm{ml}$ in the drinking water).

Histology. After survivals of 3-43 d, 55 animals with mouse donor cells (Table 1) were killed under deep terminal pentobarbitone anesthesia (Sagatal, RMB, Dagenham, UK) by transcardiac perfusion of about $200 \mathrm{ml}$ of phosphate-buffered saline (PBS). The brains were removed and rapidly frozen in crushed diy ice. Ten-micrometer cryostat sections were cut in a horizontal plane parallel to the ventral surface of the brain and dried onto gelatin-coated slides.

M6 immunohistochemistry. For M6 immunohistochemistry, sections were fixed on the slide in $4 \%$ paraformaldehyde in $0.1 \mathrm{M}$ PBS at room temperature for $20-30 \mathrm{~min}$, washed in several changes of $0.1 \mathrm{M}$ PBS for at least $30 \mathrm{~min}$, incubated for $30 \mathrm{~min}$ in $1 \%$ dried milk (as a source of nonspecific protein) in $0.1 \mathrm{M}$ PBS followed by an overnight incubation 
Table 1. The numbers of animals and the ranges of survival times for the different types and ages of donor tissue cells in the different host tracts

\begin{tabular}{llcc} 
Donor tissue & Host tract & $\begin{array}{l}\text { No. Sur- } \\
\text { of } \\
\text { ani- range } \\
\text { mals (d) }\end{array}$ \\
\hline E14/E18 neocortex & Fimbria & 10 & $6-41$ \\
E14/E18 hippocampus & Corpus callosum/cingulum & 6 & $3-36$ \\
E14 superior colliculus & Corpus callosum/cingulum & 9 & $6-43$ \\
E18 superior colliculus & Fimbria & 12 & $7-36$ \\
& Corpus callosum/cingulum & 6 & $6-31$ \\
Total & Corpus callosum/cingulum & 8 & $6-10$ \\
& & 5 & $3-7$
\end{tabular}

with a 1:30 dilution of M6 antibody (Lund et al., 1985) in 1\% dried milk in PBS at $4^{\circ} \mathrm{C}$ in a humidified chamber, washed thoroughly for 30 $\mathrm{min}$ in several changes of $0.1 \mathrm{M}$ PBS, incubated for $2 \mathrm{hr}$ in 1:100 antirat HRP-conjugated secondary $\mathrm{F}\left(\mathrm{ab}^{\prime}\right)_{2}$ antibody (Amersham, Bucks, UK) in $1 \%$ dried milk in $0.1 \mathrm{M}$ PBS at room temperature, washed thoroughly for $30 \mathrm{~min}$ in several changes of $0.1 \mathrm{M}$ PBS, incubated in $50 \mathrm{mg} / 100 \mathrm{ml}$ diaminobenzidine (DAB) and $0.006 \%$ hydrogen peroxide in phosphate buffer with $10 \mathrm{~mm}$ imidazole at pH 5.8 for $3 \mathrm{~min}$, washed thoroughly for $30 \mathrm{~min}$ in several changes of $0.1 \mathrm{M}$ PBS, then distilled water, for 30-60 min, silver intensified using a physical development (Woodhams et al., 1989), dehydrated, cleared with Histoclear (National Diagnostics, Aylesbury, UK), and mounted in a mixture of dibutyl phthalate with polystyrene and Histoclear. Some sections were counterstained lightly with aqueous thionin.

Glial fibrillary acidic protein and vimentin immunohistochemistry. For glial fibrillary acidic protein (GFAP) and vimentin immunohistochemistry, adjacent sections were either fixed with paraformaldehyde (as above) for GFAP, or fixed with 5\% acetic acid in $96 \%$ ethanol for 15 min for GFAP or vimentin. The primary antibodies were monoclonal anti-GFAP antibody (Amersham) at 1:1000, and monoclonal anti-vimentin IgG (Amersham) at 1:100, respectively. The secondary antibodies and subsequent processing were, for GFAP, 1:100 HRP-conjugated sheep anti-mouse IgG (Amersham) for $2 \mathrm{hr}$ at room temperature in $1 \%$ dried milk in $0.1 \mathrm{M}$ PBS, washed, DAB treated; for vimentin, 1:300 biotinylated horse anti-mouse IgG (Vector) in $0.1 \mathrm{~m}$ PBS for 30 min, washed, and treated with $\mathrm{ABC}$ Vectastain (Vector). All sections were washed, DAB treated, silver intensified, and lightly counterstained with aqueous thionin.

Staining host axons. For staining host axons, brains were fixed by immersion in Carnoy's fluid and embedded in paraffin wax, and $10 \mu \mathrm{m}$ sections were stained with silver (Palmgren, 1948).

\section{Results}

Suspensions of mouse donor cells from E14 or E18 hippocampus or neocortex, or from E14 superior colliculus survived well in each of the three rat host sites examined (Table 1). The transplanted cells formed compact masses, which were elongated along the axis of the host tract. All these types of donor tissue contained neurons that grew axons in each of the host tracts. E18 superior collicular cells survived poorly, and did not produce axons.

\section{M6 immunohistochemistry}

Time course. At $3 \mathrm{~d}$ after operation (the earliest time examined), the transplants and their projection fibers were M6 positive. The intensity of M6 immunoreactivity increased to a maximum by about $6 \mathrm{~d}$. The hippocampal and neocortical transplants remained M6 positive for about 6-7 weeks, after which time the intensity of M6 immunoreactivity was decreased, eventually becoming virtually undetectable. In the case of the E14 superior
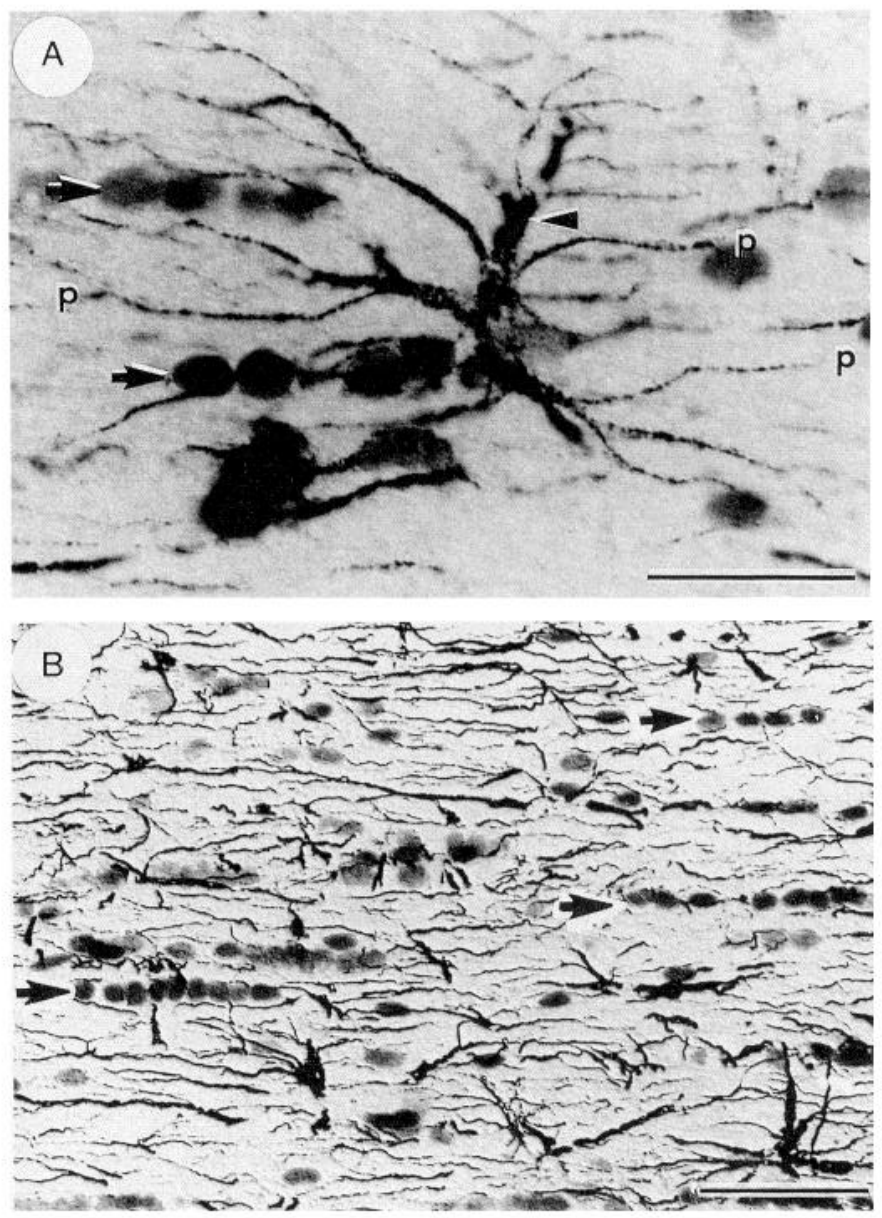

Figure 1. A, A single vimentin-positive fimbrial astrocyte (through focus double exposure), with radial process (arrowhead) and many uniform, untapering, fine longitudinal processes (e.g., p); arrows, rows of interfascicular glial nuclei. $B$, Lower-power view (GFAP staining, acid alcohol fixation, interference contrast) to show the parallel arrays of longitudinal, fimbrial astrocytic processes and interfascicular glial rows (arrows). Scale bars: $A, 20 \mu \mathrm{m} ; B, 50 \mu \mathrm{m}$.

collicular transplants, M6 immunoreactivity was downregulated by 3 weeks, that is, 3-4 weeks earlier than with the other donor tissue types.

Morphology of transplant-to-host axonal projections. The M6immunoreactive axons were of a uniform diameter of less than



Figure 2. A schematic representation of the distribution of projections (arrows) from an intrafimbrial transplant. $\mathrm{CH}$, contralateral hippocampus; $f x$, fornix column; $I H$, ipsilateral hippocampus; $L S N$, lateral septal nucleus; TSN, triangular septal nucleus; $V H C$, ventral hippocampal commissure. 

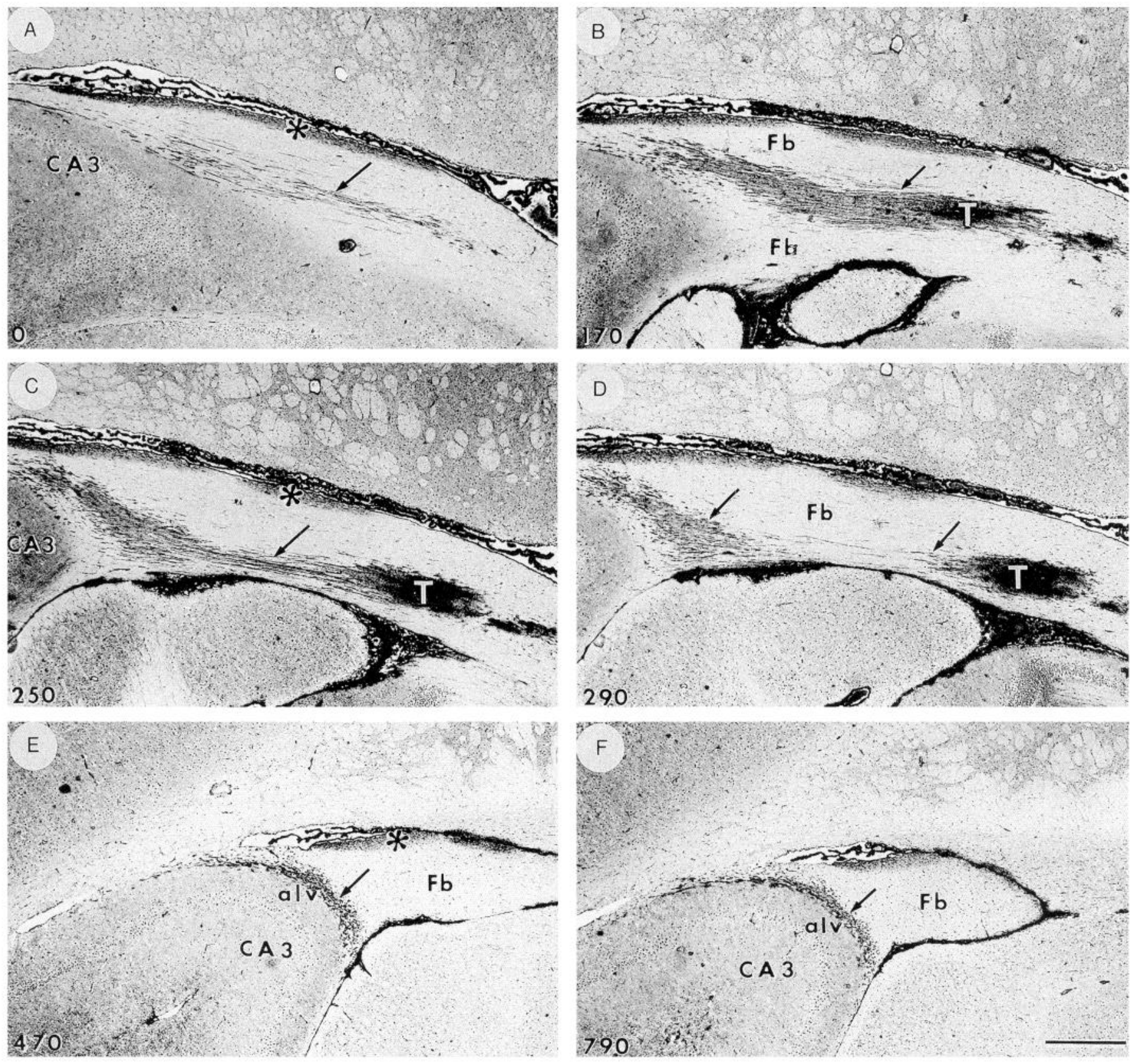

Figure 3. $A-F$, A series of six horizontal sections (from dorsal to ventral) showing an E18 cortical transplant $(T)$ generating a major laterally directed projection (arrows) through the fimbria $(F b)$ to the alveus (alv) over the lateral pole of the hippocampal field CA3. Asterisk, Staining beneath the ventricular ependyma of the lateral ventricle is nonspecific (appears with secondary anti-rat IgG alone), probably due to a regional inflammatory response. The numbers in the bottom left corner of each panel are the vertical distances in micrometers from the dorsalmost section. Survival, $6 \mathrm{~d}$. Scale bar, $500 \mu \mathrm{m}$.

$1 \mu \mathrm{m}$, and emerged from the transplants singly or in fascicles that gradually separated into individual axons. The donor axons followed the axis of the host tracts, and in places gave rise to collaterals at right angles running toward neuropil areas.

There were two patterns of staining whose distribution indicated the formation of terminal fields. These consisted of (1) branched "preterminal" axons surrounded by an area of fine staining (especially seen in the stratum oriens of the hippocampus), and (2) amorphous finely granular deposits (e.g., in the septal nuclei or the cingulate cortex).

Astrocytic markers (GFAP and vimentin). GFAP and vimentin both showed astrocytes, but with different distributions, de- pending on fixation. With aldehyde fixation, GFAP immunoreactivity was present in astrocytes in the neuropil of both host and transplant, but less in tract astrocytes. Acid alcohol fixation gave a converse pattern: both GFAP and vimentin immunoreactivity were present in the host tract astrocytes, and absent in the neuropil astrocytes.

Acid alcohol-fixed material (Fig. 1) showed the radial and longitudinal processes of the host tract astrocytes as described by Suzuki and Raisman (1992). The most striking feature was the regular array of fine parallel longitudinal astrocytic processes that became visible when the plane of section passed through the longitudinal axis of the host tract. As will be described in 

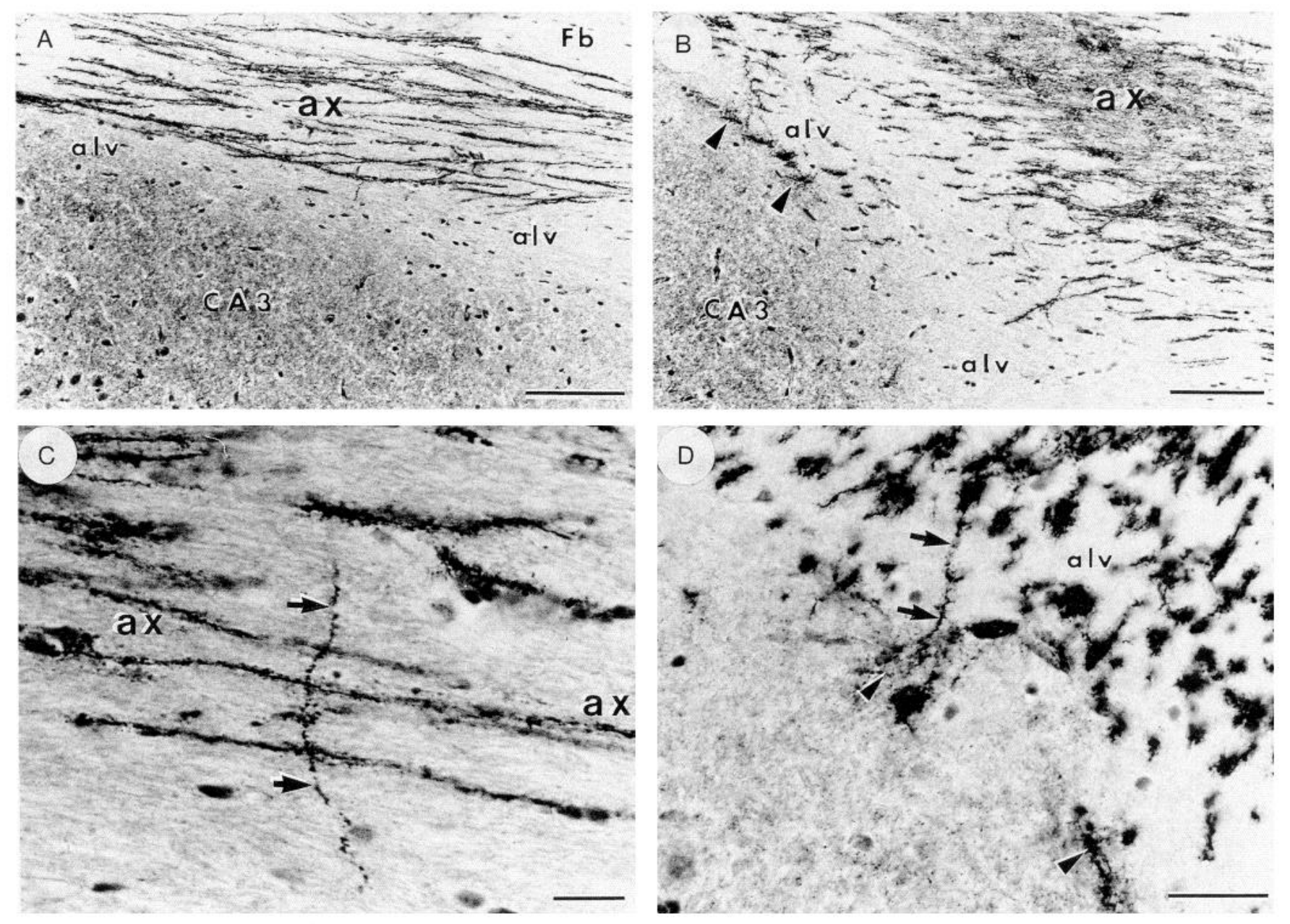

Figure 4. A, Enlarged view at the level of Figure $3 A$ to show a horizontally orientated, loose fascicle of M6-positive E18 cortical axons (ax) in the fimbria $(F b) . B$, Horizontal section through the lateral end of a horizontally running intrafimbrial bundle of axons ( $a x)$ at the level (shown in Fig. $3 C$ ) where they are beginning to turn ventrally into the alveus ( $a l v)$; arrowheads, terminal fields in the stratum oriens. $C$, A prominent collateral (arrows) in a fascicle of axons $(a x)$ such as is illustrated in $A$. $D$, Section from a level ventral to $B$ (compare Fig. $3 E, F$ ) showing ventrally directed axons in the alveus (alv) cut end-on, and a prominent collateral (arrows) leading to the upper of the two terminal fields in the stratum oriens (arrowheads). E18 cortical tissue. Survival, $6 \mathrm{~d}$. Scale bars: $A$ and $B, 100 \mu \mathrm{m} ; C$ and $D, 25 \mu \mathrm{m}$.

the different situations (see below), the donor M6-positive axons always followed a course parallel to the astrocytic longitudinal processes.

\section{Projections from transplants in the fimbria}

Hippocampal donor cells. In the previous publication (Davies et al., 1993) we demonstrated that embryonic mouse hippocampal cells transplanted into an adult rat host fimbria generated axons that projected in both directions along the longitudinal axis of the tract, that is, laterally to the ipsilateral hippocampus, medially to the septal nuclei and postcommissural fornix, and across the midline in the ventral hippocampal commissure to the contralateral hippocampus (Fig. 2). Axon collateral branches were emitted at right angles to the main axonal stems, especially where the projection skirted the hippocampal field CA3.

Cortical and superior collicular donor cells. The data from our previous study (Davies et al., 1993) were derived from transplants of hippocampal donor neurons, whose axons normally travel in the fimbria. In the present study we have transplanted "mismatched" neocortical and superior collicular donor cells whose axons never normally pass through the fimbria. Surprisingly, both E14 and E18 neocortical cells ( $n=10$ cases) and
E14 superior collicular cells ( $n=12$ cases) generated patterns of projection indistinguishable from the hippocampal donor cells ( $n=22$ cases from Davies et al., 1993).

Pattern of projection through the fimbria. The serial horizontal sections demonstrated the topographical course of the projections from transplants of E14 or E18 cortical tissue (Fig. 3). In sections dorsal to the transplant (Fig. $3 \mathrm{~A}$ ), the fibers were cut in their lateral course as they arched upward from the transplant, running toward the ipsilateral host hippocampus. At progressively more ventral levels (Fig. $3 B-D$ ), the fibers fanned out into the alveus (Fig. $3 E, F$ ) over the lateral pole of the hippocampal field CA3, becoming progressively more vertical as they followed the curve of the hippocampus. Along the whole of this interface, the donor projection fibers turned into the underlying stratum oriens (Fig. 4), giving rise to prominent collaterals (Fig. $4 C, D)$ and diffuse staining, suggesting the formation of terminal fields (Fig. 4B,D). The same pattern, extent, and density of projections were produced by transplants of E14 superior collicular tissue (Fig. 5).

Rostromedially from both cortical and collicular transplants, the emerging fibers (RM in Fig. 6; cf. Fig. $6 B$ in Davies et al., 1993 ) entered the septal nuclei and produced a diffuse, terminal 

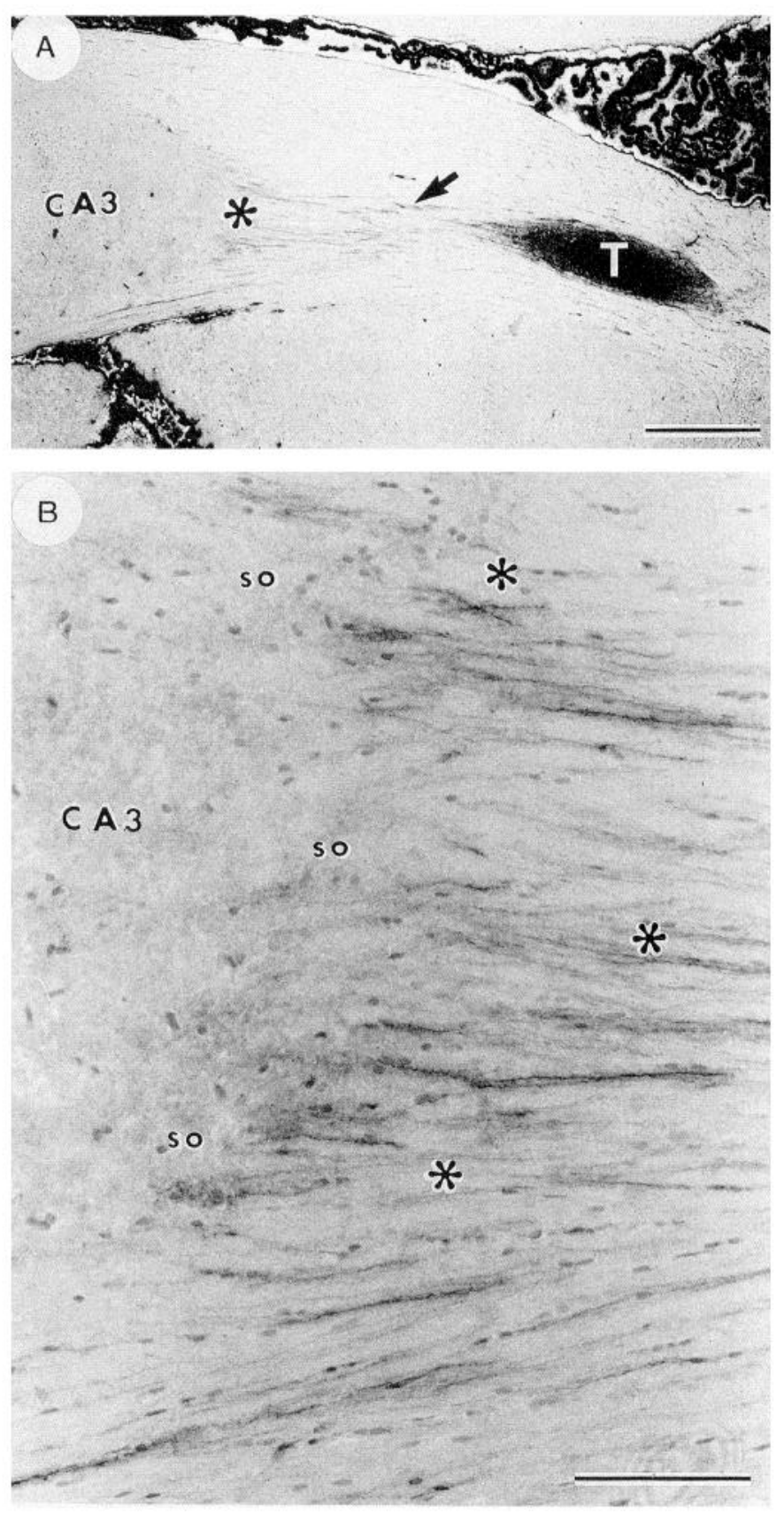

Figure 5. A, An E14 superior collicular transplant $(T)$ generating a laterally directed intrafimbrial projection (arrow) that reaches the alveus of the hippocampal field CA3 (asterisk, region enlarged in $B$ ). B, Preterminal M6-positive projection fibers traverse the alveus (asterisks) to produce terminal field-type staining in the stratum oriens (so). Survival, $16 \mathrm{~d}$. Scale bars: $A, 500 \mu \mathrm{m} ; B, 100 \mu \mathrm{m}$.

field type of staining (Fig. 7), which was no less dense or extensive than that produced by transplants of the appropriate, hippocampal cells. In the more ventral sections isolated individual M6-positive donor fibers entered the host postcommissural fornix columns where they were cut, like the host fornix fibers, end-on (solid arrow in Fig. $7 B$ ). Caudal to this a contingent of fibers (CM in Fig. 6) left the transplant to enter the ventral hippocampal commissure and cross the midline into the contralateral fimbria (Fig. 8).

Effect of location in the fimbria. Smaller intrafimbrial trans-

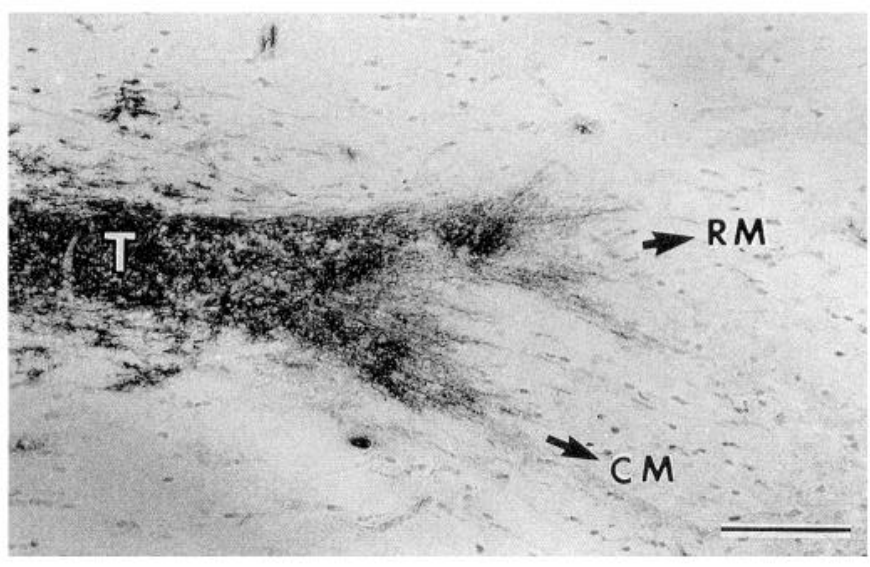

Figure 6. Medial edge of an intrafimbrial E18 cortical transplant $(T)$ generating rostromedially $(R M)$ and caudomedially $(C M)$ directed streams of axons (arrows) toward the septal neuropil and ventral hippocampal commissure, respectively. Survival, $34 \mathrm{~d}$. Scale bar, $100 \mu \mathrm{m}$.

plants produced less extensive projections, which varied according to the location of the transplants in the fimbria. Three individual cases exemplify these "partial" patterns.

(1) A transplant in the caudal part of the fimbria at the level of the ventral hippocampal commissure gave rise to a "beam" of fibers that remained confined to the caudal fimbria and ran directly into the alveus over the medial part of field CA3 (Fig. 9A).

(2) A small transplant of hippocampal cells (taken from the series in Davies et al., 1993) lay farther back and laterally at the level of the hippocampal flexure. This transplant straddled the boundary between the fimbria and stria terminalis, with some donor cells in both tracts. The transplanted cells gave rise to two narrow beams of axons, one in the fimbria and one in the stria terminalis (Fig. $9 B$ ).

(3) A transplant lying in the dorsomedial edge of the fimbria did not send any fibers laterally in the fimbria, but did project medially, to produce a large area of terminal field-type staining in the immediately adjacent lateral septal nucleus (Fig. 9C).

Correlation of donor axon projections and the orientation of the host tract structures. The present material consistently confirmed our previous finding (Davies et al., 1993) that the orientation of the M6-positive donor axons (e.g., arrows in Fig. $10 \mathrm{~A}$ ) is parallel to that of the host fimbrial axons (arrowheads in Fig. 10A) and to the interfascicular rows of glial cell bodies (igr in Fig. 10A). Where the longitudinal structures of the host tract were cut end-on (e.g., arrows in Fig. 10B), the donor axons were also cut end-on (ax in Fig. 10B).

A prominent element in the fimbrial architecture is the parallel array of longitudinal astrocytic processes along the axonal axis of the tract (Fig. 1; Suzuki and Raisman, 1992). Using the vimentin immunoreactivity of these processes in the fimbria, we have been able to show that the pathway taken by the donor axons strictly follows the orientation of the host longitudinal astrocytic tract processes (Figs. 11, 12).

In contrast, the astrocytes of the transplant neuropil are vimentin negative (Fig. $11 B$ ), but they can be stained with GFAP, which shows that along the transplant/host interface, the processes of the astrocytes in the transplant neuropil are orientated such that they flow out smoothly and merge with the plane of orientation of the surrounding host tract longitudinal astrocytic 

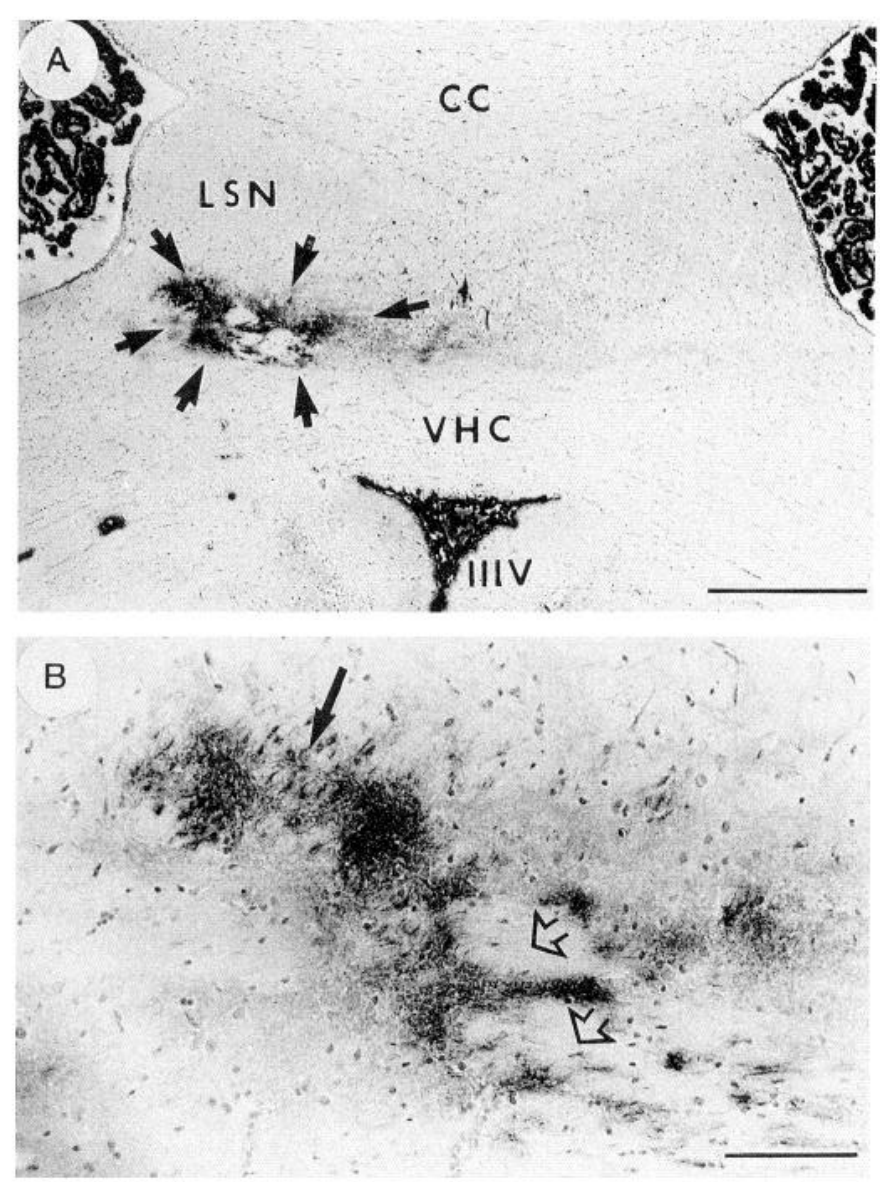

Figure 7. A, Diffuse terminal field-like staining (arrows) in the caudal part of the lateral septal nucleus $(L S N)$; $C C$, corpus callosum; $V H C$, ventral hippocampal commissure; $I I I V$, third ventricle. $B$, Enlarged view of part of the terminal field in $A$, showing adjacent clear areas occupied by the host fornix bundles (open arrows). Solid arrow shows a fornix bundle containing scattered M6-positive transplant axons cut end-on. E14 superior collicular transplant. Survival, 14 d. Scale bars: $A, 500 \mu \mathrm{m} ; B, 100 \mu \mathrm{m}$.

processes (illustrated for an intracingulate transplant in Fig. 24, below).

In the positions where the M6-positive axons ran laterally in the fimbria, there was a matching parallel stretch of longitudinal astrocytic processes (Fig. 11). Farther laterally, where the transplant axons (Figs. 10B, 12A) turned ventrally through the plane of the section (and were therefore cut end-on), the inclination of the vimentin-positive host longitudinal astrocytic processes (Fig. 12B) and the rows of host interfascicular glial nuclei (Fig. $10 B$ ) changed exactly in parallel, such that the M6-positive donor axons, the host interfascicular glial nuclei, and the host astrocytic longitudinal processes were all cut end-on.

\section{Projections from transplants in the corpus callosum and cingulum}

Embryonic donor cells injected into the corpus callosum and/ or cingulum generated long interfascicular axon projections. The patterns of distribution of the donor projections were the same for E14 or E18 hippocampal ( $n=9$ cases) or cortical ( $n=6$ cases) cells, or for E14 superior collicular cells ( $n=6$ cases); however, as in the fimbria, the M6 immunoreactivity consistently disappeared from the superior collicular axons much earlier than from the hippocampal or neocortical axons.

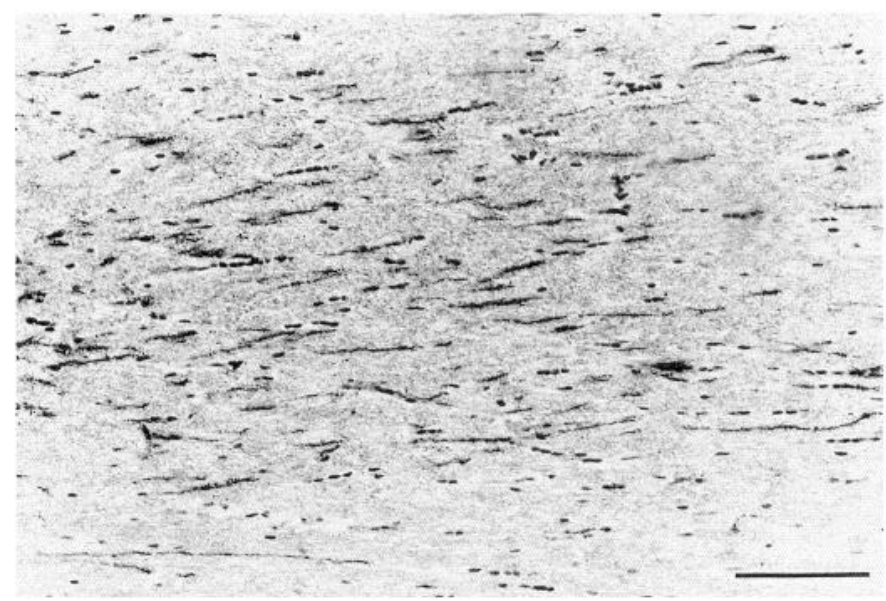

Figure 8. Scattered M6-positive axons in the contralateral fimbria; generated by an E18 cortical transplant in the ipsilateral fimbria. Survival, $6 \mathrm{~d}$. Scale bar, $100 \mu \mathrm{m}$.

Intracallosal transplants. The transplants were placed in the rostrum of the corpus callosum, about $1.0-1.2 \mathrm{~mm}$ lateral to the midline. The overall pattern of the projections is shown at nine representative horizontal levels in Figure 13.

Fibers emerging from the dorsal part of the transplants radiated out through the callosum dorsally, laterally, and rostrally into the cortex (Figs. $13 A, B ; 14$ ). In the horizontal plane of section, these radiating fibers were cut in short segments, with numerous collaterals arising from the main axonal stems, and many bifurcations as the fibers formed an increasingly complex plexus at the entry to gray matter of the overlying cortex.

Throughout the approximately $300 \mu \mathrm{m}$ of horizontal distance represented by levels $C$ and $D$ of Figure 13, no projections emerged from the transplants in the horizontal plane of section. (As will be seen below, this correlates with the orientation pattern of the host tract astrocytic processes.)

At more ventral levels (Fig. 13E-I) major projections arose from the medial surface of the transplant. The most dorsal of these projection fibers ran medially into the gray matter of the cingulate cortex and generated a diffuse-type terminal field (asterisks in Fig. 15). The majority of the medially directed fibers took a downward course to mingle with the midline-crossing fibers of the callosum (Fig. 16), where they formed characteristic, rather coarse, interweaving fascicles, exactly matching the pattern of the host callosal axons (Fig. 17). Once they had crossed the midline, the fibers took a course (ascending laterally and rostrally in the contralateral hemisphere) that was a mirror image of their previous trajectory through the ipsilateral hemisphere (asterisk in Fig. 13F). Collaterals arising from these axons were directed radially into the contralateral cerebral cortex.

Intracingulate transplants. Cell suspensions from each of the three types of donor tissue were injected into the cingulum in a position slightly medial to the intracallosal transplants. The difference from the intracallosal transplants was striking: whereas the intracallosal transplants generated transversely orientated fibers in conformity with the transverse orientation of the host callosal axons, in contrast the intracingulate transplants generated longitudinally orientated fibers, in conformity with the longitudinal orientation of the host cingulate fibers (Fig. 18 illustrates a transplant making both types of projection).

Long fiber projections arising from the intracingulate transplants ran in a rostrocaudal orientation within the bundles of 



Figure 10. A, A long M6-positive donor axon (arrows) lying parallel to the host fimbrial axons (e.g., arrowheads) in the contralateral fimbria. $i g r$, row of interfascicular glial nuclei lying in the plane of section. $B$ Alvear region where the M6-positive donor axons ( $a x)$ are cut end-on, and the interfascicular glial rows are also cut end-on, and are therefore represented by single nuclei (e.g., arrows). E1 8 cortical transplants. Survival, $36 \mathrm{~d}(A), 6 \mathrm{~d}(B)$. Scale bars: $A, 20 \mu \mathrm{m} ; B, 50 \mu \mathrm{m}$.

host cingulate fibers (Fig. 18), and gave rise to diffuse-type terminal field staining in the gray matter of the cingulate gyrus (Fig. 19).

With all three donor cell types, a consistent peculiarity of the intracingulate grafts was a characteristic, narrow M6-positive column, about $50-100 \mu \mathrm{m}$ wide (see below in Fig. 23), which passed radially for $1 \mathrm{~mm}$ or more through the gray matter of the cingulate gyrus, in a direction rostrally and dorsally at an angle to the injection track.

The Palmgren-stained material showed that there was no in-

Figure 9. A, A small E14 superior collicular transplant $(T)$ in the caudal edge of the fimbria $(F b)$ generates an M6-positive projection (arrow) that remains confined to the caudal margin of the fimbria (compare Fig. 3 ) and runs directly into the alveus of the adjacent part of field CA3 (arrowhead). B, Narrow, aligned beam of axons (arrows) in the stria terminalis $(S T)$ and fimbria $(F b)$, derived from an E18 hippocampal transplant (out of the plane of section) that straddles the junction of the tracts. C, An E14 superior collicular transplant ( $T$ ) partially surrounding the host fornix columns (asterisks) into which the transplant contributes M6-positive fibers at a more ventral level (as at solid arrow in Fig. 7B). This transplant generates terminal field-type diffuse staining in the adjacent part of the host lateral septal nucleus (arrow), but does not project laterally into the host fimbria $(F b)$. Survival, $7 \mathrm{~d}$. Scale bars: $A, 500$ $\mu \mathrm{m} ; B, 200 \mu \mathrm{m} ; C, 100 \mu \mathrm{m}$ terchange of host fibers between the corpus callosum and the cingulum at the level we used for transplantation (Fig. 20A). When a transplant was confined to one or another tract, the transplant axonal projections were also confined to the same tract (Fig. 20B,C). Transplants that straddled the border of the callosum and cingulum (such that some donor cells lay in each of the tracts; boxed area in Fig. 20) gave rise to axonal projections that followed both tracts (Figs. 18, 19, 20D).

Correlation of donor axon projections and the orientation of the host tract structures. As with the intrafimbrial transplants, the donor axons in the corpus callosum and cingulum were parallel to the host axons, to the rows of interfascicular glial cell bodies, and to the vimentin-positive longitudinal processes of the host tract astrocytes (e.g., compare Figs. 11, 22). 


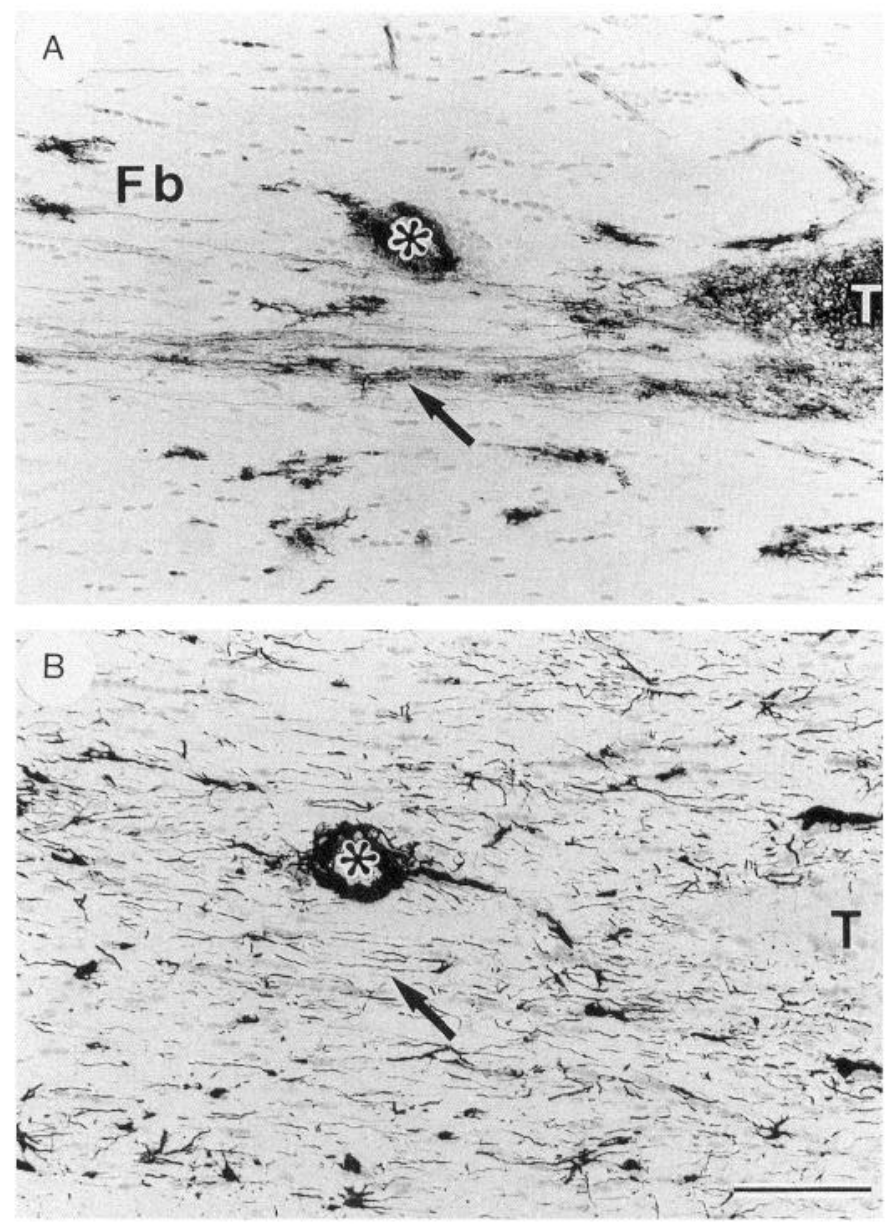

Figure 11. A, Lateral edge of an E18 cortical transplant ( $T$ ) generating a long M6-positive fiber projection (arrow) into the fimbria $(F b) . B$, The same area (located by the position of the large blood vessel, asterisk) from an adjacent section to show vimentin-positive longitudinal astrocytic processes (e.g., at arrow) orientated in parallel with the transplant axons. Note that the astrocytes in the transplant neuropil $(T)$ are vimentin negative. Survival, $34 \mathrm{~d}$. Scale bar, $100 \mu \mathrm{m}$.

Thus, (1) from the dorsal parts of the transplants the host callosal astrocytic longitudinal processes lay in line with the M6positive corticopetal projection fibers leaving the transplant.

(2) In sections through the intermediate dorsoventral course of the transplants at levels (Fig. 13C,D) where no fibers emerged in the plane of section, the longitudinal astrocytic processes of the host tract were cut end-on (Fig. 21).

(3) From the ventral parts of the transplants, the longitudinal astrocytic processes paralleled the donor axons on their way to cross the midline (Fig. 22).

(4) The M6-positive column (Fig. 23A,C) through the gray matter of the cingulate gyrus was intensely vimentin positive (Fig. 23B,D), and stood out against the adjacent vimentin-negative host gray matter of the cortex. This was the only situation in which we observed vimentin induction in gray matter. The column was cut transverse to its long axis, and both the M6positive transplant projection fibers and the host vimentin-positive longitudinal astrocytic processes were also orientated endon to the plane of section.

$G F A P$. As with the intrafimbrial transplants, the GFAP-stained astrocytic processes of the intracallosal and intracingulate transplants ran in continuity from the interior of the transplant to
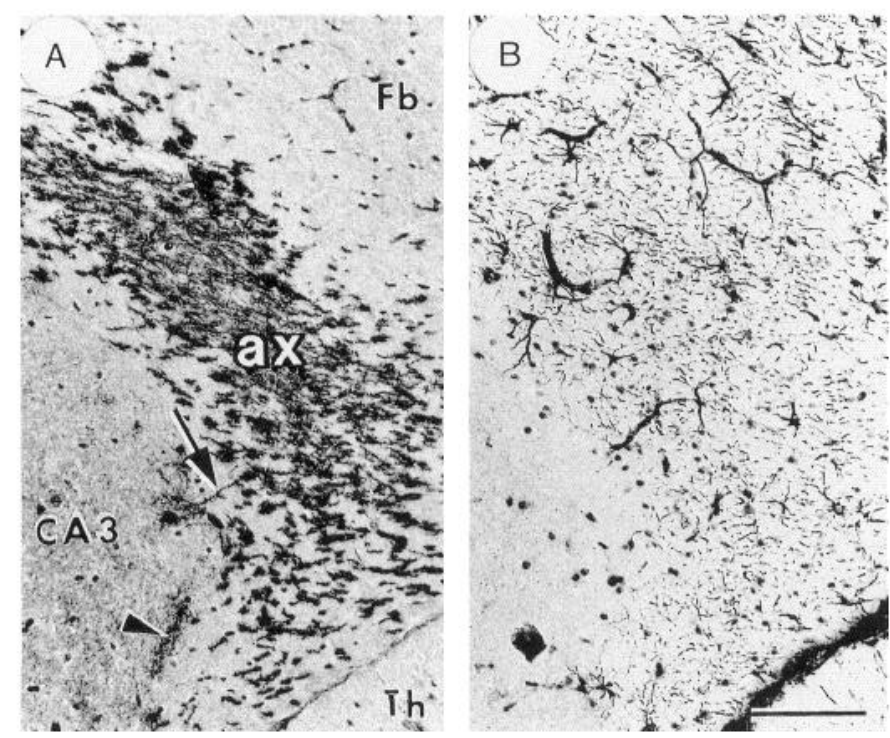

Figure 12. A, Crescent-shaped area of M6-positive axons (ax) in the alveus over the medial pole of the hippocampal field CA3 at the levels shown in Figure 3, $C$ and $D$. These axons are seen in an almost endon view as they run vertically through the horizontal plane of the section (as in Fig. 10B). Arrow, collateral; arrowhead, terminal field in the stratum oriens; $F b$, fimbria; $T h$, thalamus. $B$, Adjacent section stained for vimentin to show the predominantly end-on views (compare Fig. 10B) of the fimbrial longitudinal astrocytic processes that are parallel to the M6-positive axons. E18 cortical transplant. Survival, 6 d. Scale bar, $100 \mu \mathrm{m}$.

become confluent with the adjacent vimentin-stained longitudinal astroglial processes of the host tract astrocytes (Figs. $21 C$, 24).

\section{Distance and speed of long interfascicular axon growth}

We have previously described the rate of growth of axons from embryonic hippocampal neurons transplanted into the fimbria (Davies et al., 1993). In this situation, the axons were present by $3 \mathrm{~d}$, and extended at least $1 \mathrm{~mm} / \mathrm{d}$, such that they reached their furthest targets (on the contralateral side) at a distance of about $10 \mathrm{~mm}$ by $1-2$ weeks.

In the present material we found a comparable speed and distance of growth of axons from each of the different viable donor cell types in each of the host tracts examined. Thus, the axons from neocortical and superior collicular cell grafts traversed the host fimbria at the same rate as the axons from donor hippocampal cells, reaching the ipsilateral hippocampus and contralateral fimbria by $6 \mathrm{~d}$. At the same survival time, projection fibers from intracallosal transplants of all three types of donor cells had already traversed the midline and started to ramify in the contralateral cortex.

\section{Discussion}

\section{Long interfascicular axon growth}

It has long been thought that fiber growth in adult brain is restricted to short distances, and only occurs in neuropil. Recently, however, retrograde cell labeling techniques have shown that transplanted embryonic neurons are able to establish connections with distant target regions in adult brain (Tønder et al., 1990) and, as in the present study, direct visualization of donor nerve fibers shows that the axons of embryonic neurons can grow for surprisingly long distances in adult host myelinated 

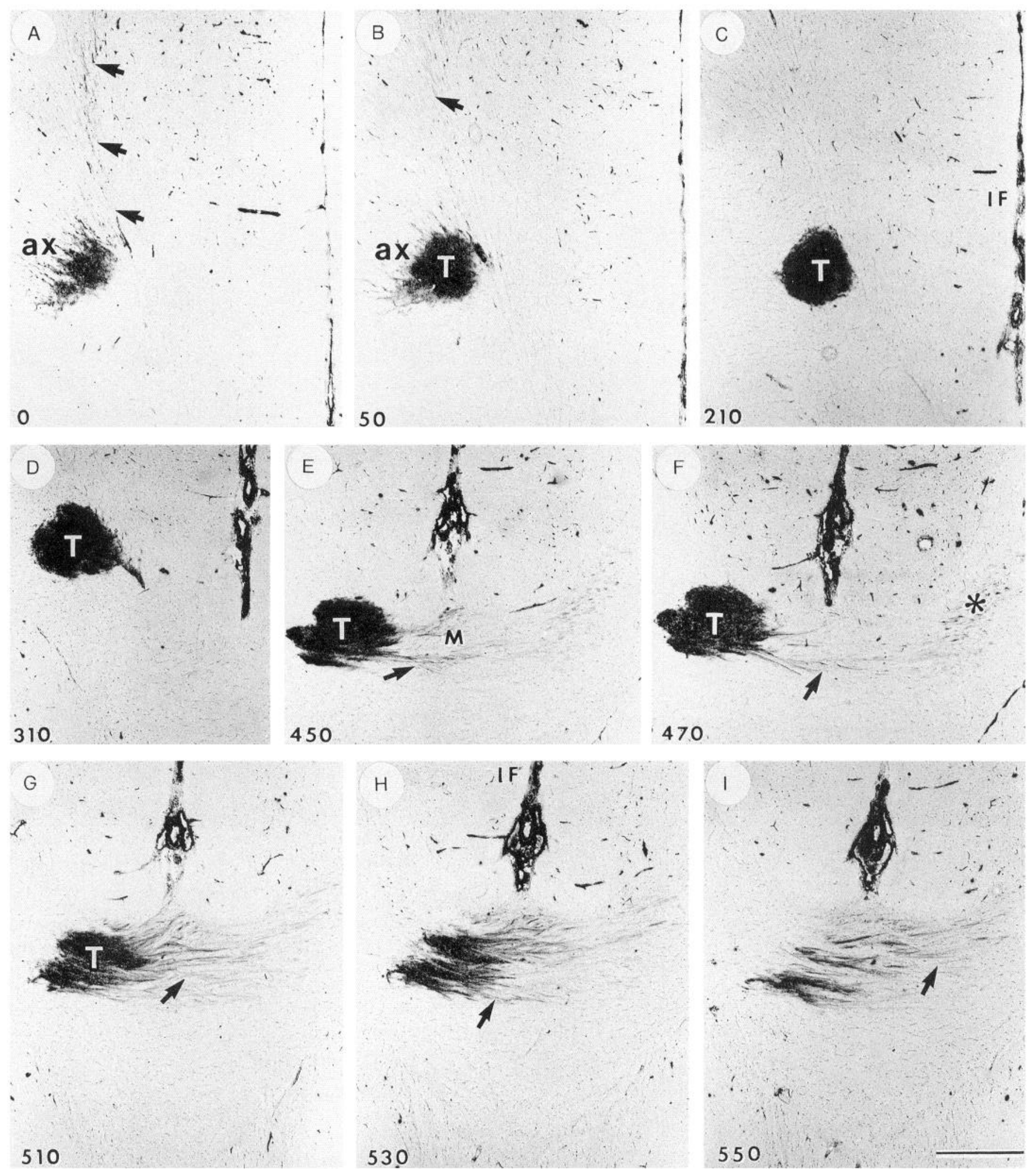

Figure 13. A-I, Series of horizontal sections from dorsal to ventral through an E14 superior collicular transplant $(T)$ in the rostrum of the corpus callosum. $A$ and $B$, Levels with M6-positive axons $(a x)$ radiating from the rostrolateral aspect of the transplant. Arrows show projection into the cingulum. $C$ and $D$, Intermediate part of the transplant with no projection fibers leaving the transplant in the plane of the section. $E-I$, Ventral levels, showing medially directed projection fibers (arrows) running across the midline $(M)$ in the corpus callosum. Asterisk in $F$, fibers that have crossed the midline and are ascending on the opposite side in a position symmetrical to the transplant. $I F$, interhemispheric midline fissure. The numbers in the bottom left corners of each panel are the vertical distances in micrometers from the dorsalmost section. Survival, $21 \mathrm{~d}$. Scale bar, $500 \mu \mathrm{m}$. 



Figure 14. A, Fascicles of M6-positive axons ( $a x$ ) radiating rostrally from an E14 hippocampal transplant in the rostrum of the corpus callosum. $B$, Enlarged view. Survival, $43 \mathrm{~d}$. Scale bars: $A, 100 \mu \mathrm{m} ; B, 50$ $\mu \mathrm{m}$.

fiber tracts (Fujii, 1991; Wictorin et al., 1991, 1992; Strömberg et al., 1992; Davies et al., 1993).

Most experiments have used quite large and therefore traumatic grafts of embryonic donor tissue into host neuropil areas such as the striatum (Fujii, 1991; Wictorin et al., 1991, 1992), hippocampus (Tønder et al., 1990), or cortex (Fujii, 1991; Sørensen et al., 1992; Strömberg et al., 1992). In an attempt to


Figure 15. A, Caudal edge of an E14 hippocampal transplant $(T)$ with a short bundle of M6-positive fibers (arrow) forming a terminal field (asterisk) in the gray matter of the cingulate cortex (CGyr). B, A section from a more ventral level, where the projection (arrow) to the cingulate cortex is still present, but on its lateral surface there is now a contingent of callosal fibers (arrowheads) running ventrally on their way to cross the midline. Survival, $43 \mathrm{~d}$. Scale bar, $100 \mu \mathrm{m}$.
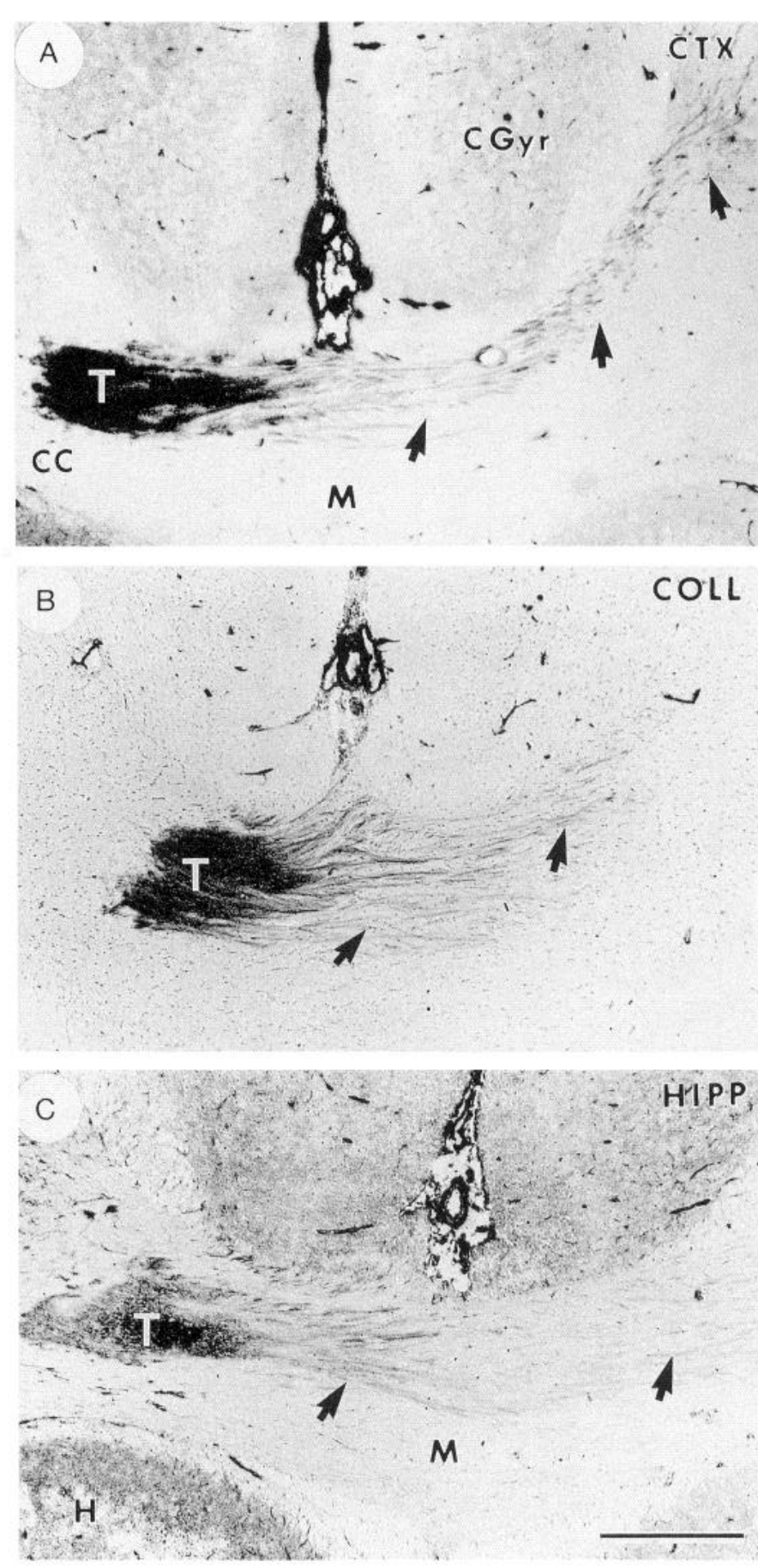

Figure 16. Midline-crossing M6-positive axonal projections (arrows) from intracallosal transplants $(T)$ of E14 cortical $(C T X$, in $A)$, E14 superior collicular (COLL in $B)$, and E14 hippocampal (HIPP in $C$ ) cells. $C C$, corpus callosum; $C G y r$, cingulate gyrus; $H$, hippocampus, $M$, midline. Survival, 7, 21, $43 \mathrm{~d}(A, B, C$, respectively). Scale bar, 500 $\mu \mathrm{m}$.

simplify the experimental design, and to concentrate on the local tissue factors present in the tracts, we have used a microtransplantation procedure (Emmett et al., 1990) to inject small numbers of embryonic cells directly into adult fiber tracts. Because these injections are relatively atraumatic, the procedure virtually eliminates scarring, and the donor neurons and glia come rapidly into contact with largely undisturbed host tract structures. 

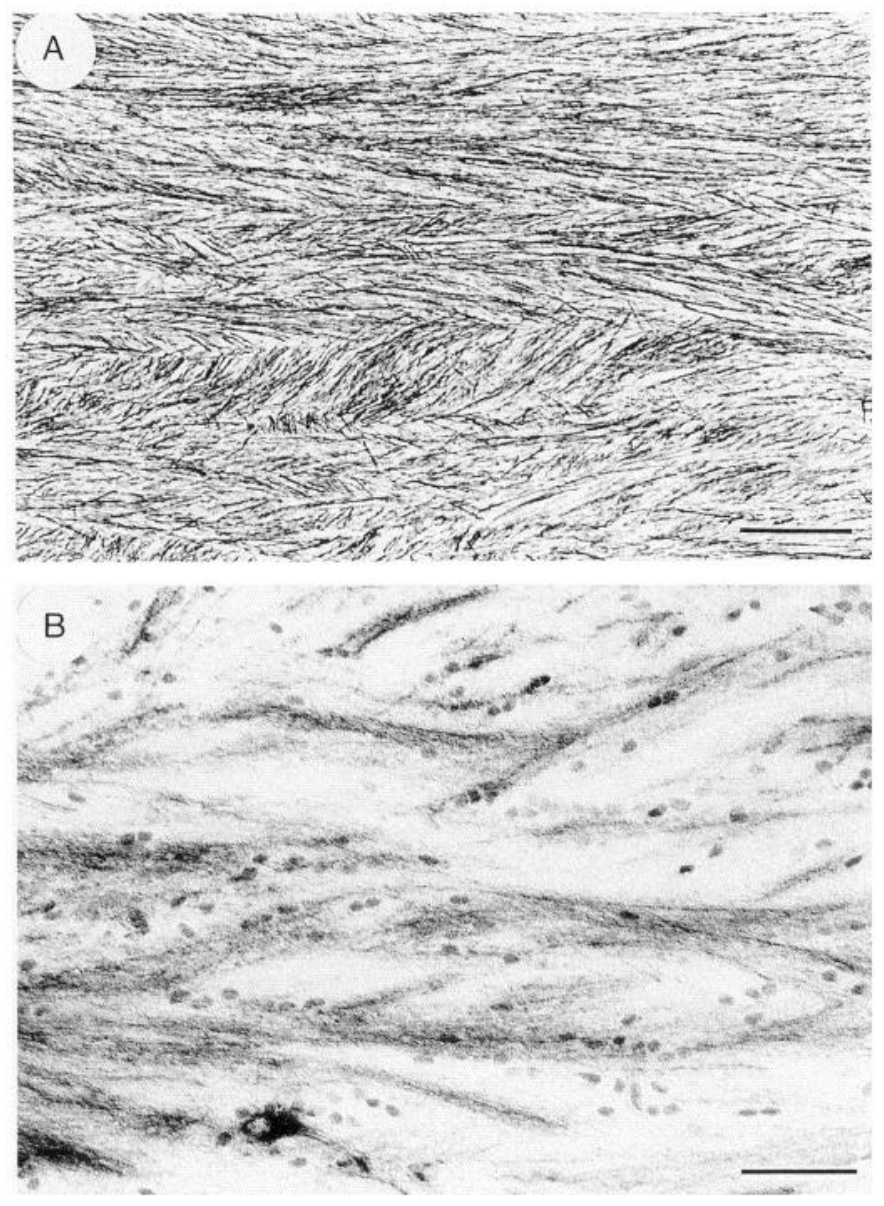

Figure 17. Interweaving of interhemispheric-crossing fibers at the midline of the corpus callosum. $A$, Host fibers (Palmgren stain) from a normal animal. $B$, Donor axons (M6 stain) from the E14 cortical transplant shown in Figure 16A. Survival, $7 \mathrm{~d}$. Scale bars: $A, 100 \mu \mathrm{m} ; B, 50$ $\mu \mathrm{m}$.

In a previous study (Davies et al., 1993) we used the mousespecific neuronal surface membrane glycoprotein markers M6 (Lund et al., 1985) and Thy-1 (Zhou et al., 1985; Fujii, 1991) to demonstrate LIAG from embryonic mouse hippocampal neurons transplanted into the adult rat fimbria (Davies et al., 1993). We now show that LIAG also occurs in the adult corpus callosum (see also Tønder et al., 1988; Fujii, 1991; Wictorin et al., 1991; Strömberg et al., 1992) and cingulum with the same vigor, speed, and efficiency as in the fimbria. In less than 2 weeks, the donor axons had traversed the full length of the fimbria and corpus callosum to reach their terminal fields in the hippocampus and cortex of the same and the opposite sides, at distances of up to about $10 \mathrm{~mm}$ (Davies et al., 1993). When transplanted into spinal cord (Li and Raisman, 1994), M6-stained hippocampal donor axons were also observed to travel for about 10 $\mathrm{mm}$ in 2-3 weeks (see also Wictorin and Björklund, 1992). Although we do not know whether the M6-labeled axons in the present study become myelinated, Wictorin et al. (1990b), using Phaseolus vulgaris leucoagglutinin (PHAL) labeling, were able to demonstrate myelination of donor axons in the internal capsule.

In all tracts we studied, the donor axons formed collaterals which arose at right angles to the main axonal stems (see also Fig. $2 c$ in Wictorin et al., 1990a), and resembled those formed



Figure 18. A, M6-positive projections from an E14 superior collicular transplant (part of which is visible at $T$ ), which straddles the corpus callosum $(C C)$ and cingulum $(\mathrm{cg})$, and gives rise to axons radiating rostrolaterally in the corpus callosum (arrow), and rostrally (boxed area shown in $B$ ) in the cingulum. $B$, Enlarged view of intracingulate fascicles boxed in $A$. Survival, $21 \mathrm{~d}$. Scale bars: $A, 500 \mu \mathrm{m} ; B, 50 \mu \mathrm{m}$.

in normal development (e.g., O'Leary and Terashima, 1988; O'Leary et al., 1990; Simon and O'Leary, 1992).

In both this and the previous publication, the light microscopic patterns of diffuse M6 immunostaining suggested that the donor axons were forming terminal fields. We have not confirmed the presence of donor synapses by electron microscopy (cf. Lund et al., 1985), but Wictorin et al. (1990b) used PHAL staining to demonstrate that interfascicular axons formed by embryonic striatal neurons transplanted into the adult striatum are able to traverse the internal capsule for $1.2 \mathrm{~mm}$ to form synaptic terminals in the globus pallidus.

\section{Why is LIAG not prevented by myelin-associated inhibitory molecules in adult host tracts?}

Much recent work has focused on the idea that the failure of central regeneration is due to the fact that mature myelinating oligodendrocytes express surface molecules that inhibit axon growth (Schnell and Schwab, 1990; Schwab et al., 1993). To reconcile the concept of an inhibitory molecule with their observation of long axon growth from embryonic donor cells along myelinated fiber tracts in the adult CNS, Wictorin and collaborators suggested that immature axons (especially from human neuroblasts) may not have the receptors to respond to the inhibitory influences present in adult myelinated tracts (Wictorin et al., 1990a, 1992). Although we have observed vigorous growth from nonhuman donor cells, in other respects our observations do not rule out this possibility.

\section{LIAG does not require correct matching of donor tissue origin to host tract}

In our previous study, we had observed the formation of axons by embryonic hippocampal neurons transplanted into the adult fimbria. Since this is the tract in which the host hippocampal 


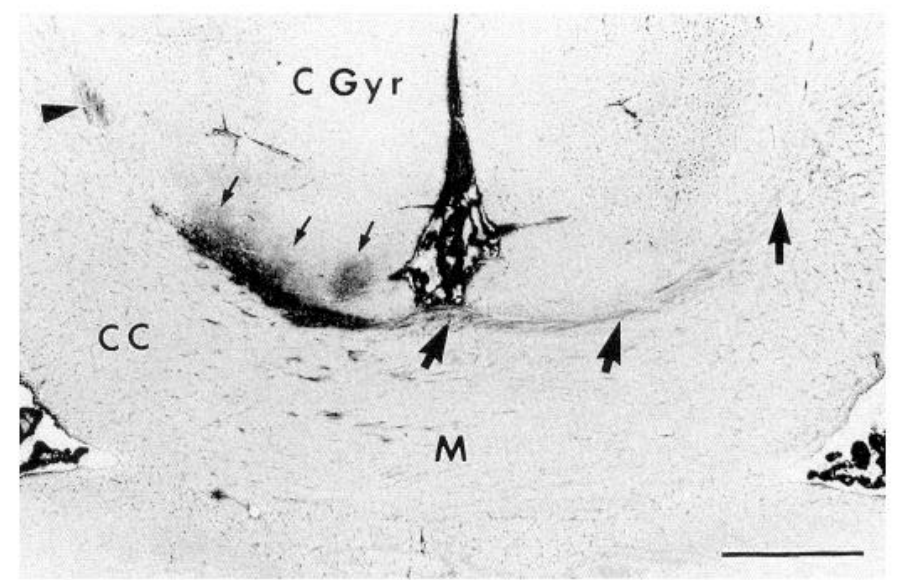

Figure 19. Distribution of projections formed by an E18 cortical transplant (the main part of which lies out of the plane of section) straddling the boundary of the corpus callosum $(C C)$ and cingulum. Small arrows, densely M6-positive, diffuse-type terminal projection in the gray matter of the cingulate cortex (CGyr); large arrows, fascicles crossing the midline $(M)$ in the corpus callosum; arrowhead, small fascicle in the cingulum bundle. Survival, $19 \mathrm{~d}$. Scale bar, $500 \mu \mathrm{m}$.

axons run, it was appropriate that the donor hippocampal axons should have reproduced the normal patterns of axon trajectory through the fimbria, and entered the same terminal fields in the hippocampus and septal nuclei as those innervated by the normal adult host hippocampal axons that make up this tract.

It was surprising, though, to find in the present study that transplanted embryonic neocortical and superior collicular donor neurons, whose axons never normally enter the fimbria, consistently generated exactly the same pattern of intrafimbrial distribution, at no lesser speed or density than hippocampal donor cells, and mimicked hippocampal donor cells even to the point of forming collaterals and entering terminal fields. Similarly, transplants of cell suspensions from all three donor regions (E14 or E18 hippocampal or neocortical donor tissue, or E14 superior collicular tissue) produced indistinguishable patterns of projection in the adult host corpus callosum and cingulum. Clearly the environment of the adult host tracts provides sufficient cues to induce axon formation from neurons that do not normally project through those specific tracts. Possibly this might be analogous to the early stage of "exuberant" axonal growth that occurs in normal in situ tract development before the time when contact with the terminal field determines the refined adult projection pattern and loss of the incorrectly matched axonal projections (see below). There are, however, other possibilities.

Our tissue samples would have contained neuroblasts as well as different types of neurons, and we do not know which of the donor cells gave rise to the interfascicular axons. It is possible that the experimental situation selects for special categories of cells (e.g., those belonging to more diffuse or "global" systems, rather than the topographically precise "point-to-point" systems; Sotelo and Alvarado-Mallart, 1987). Alternatively, as has been shown in cortical transplantation experiments (Barbe and Levitt, 1991; O'Leary and Koester, 1993), the host tissue environment may alter the differentiation and neural connections of the transplanted developing donor cells. There has been much recent interest in the possibility that the CNS may contain multipotential stem cells whose fate is determined by their environment. Thus, it has been shown that oncogene-mediated immortalization of early neuroepithelial cells can produce cell lines
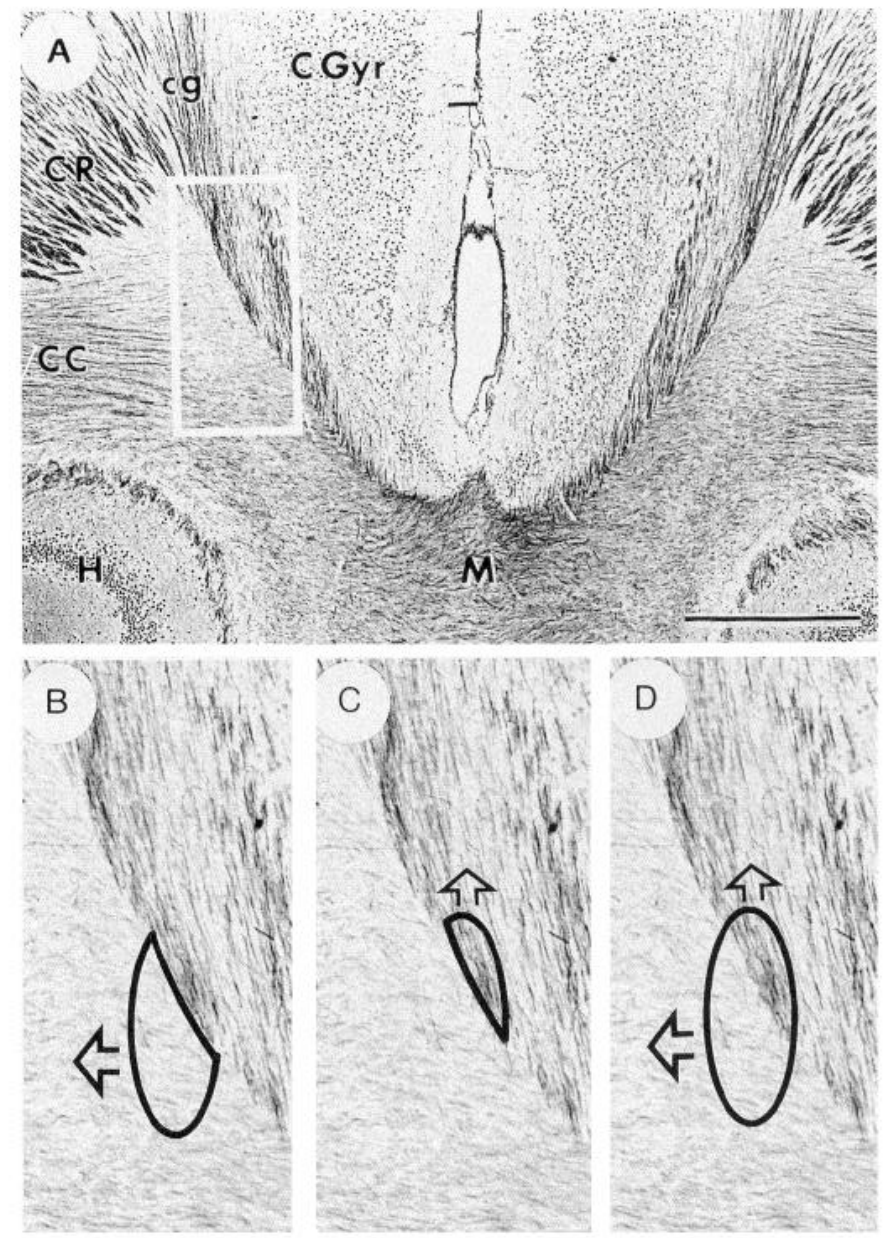

Figure 20. A, Palmgren-stained horizontal section through the rostral part of the corpus callosum to show the longitudinally arranged fibers of the cingulum $(\mathrm{cg})$ lying adjacent to the medial edge of the mass of transversely orientated fibers of the corpus callosum $(C C)$. $C G y r$, cortex of the cingulate gyrus; $C R$, corona radiata; $H$, hippocampus; $M$, midline. $B-D$, Enlarged views of the boxed area in $A$, with a schematic representation of the different types of projections (arrows) according to the exact location of the transplants at the callosal/cingulum interface. The donor axonal projections from transplants in $B$ and $C$ are restricted to the corpus callosum (larger arrows) or the cingulum (smaller arrows), respectively, and do not cross the intertract boundary. $D$, Axons from transplants straddling the boundary project into both tracts. Scale bar, $500 \mu \mathrm{m}$.

that have the potential to differentiate into different neuronal cell types characteristic of specific host regions into which they are transplanted (Renfranz et al., 1991; Snyder et al., 1992), and adult brain tissue stimulated with epidermal growth factor contains stem cells that can be induced to differentiate into different types of neurons (Reynolds and Weiss, 1992).

In contrast to the behavior of cells transplanted into adult tracts, there are a number of experimental situations in which it has been demonstrated that the formation of terminal field projections from transplants in direct contact with specifically denervated host neuropil maintains appropriate tissue-type specificity (e.g., Zhou et al., 1985, 1989; Wictorin et al., 1991, 1992), with only occasional abnormalities (e.g., Raisman and Ebner, 1983). Wictorin et al. (1991, 1992) demonstrated clear tissuetype specificity in the formation of interfascicular projections from different types of donor tissue. On the other hand, Fujii (1991) reported long interfascicular growth of axons from mis- 

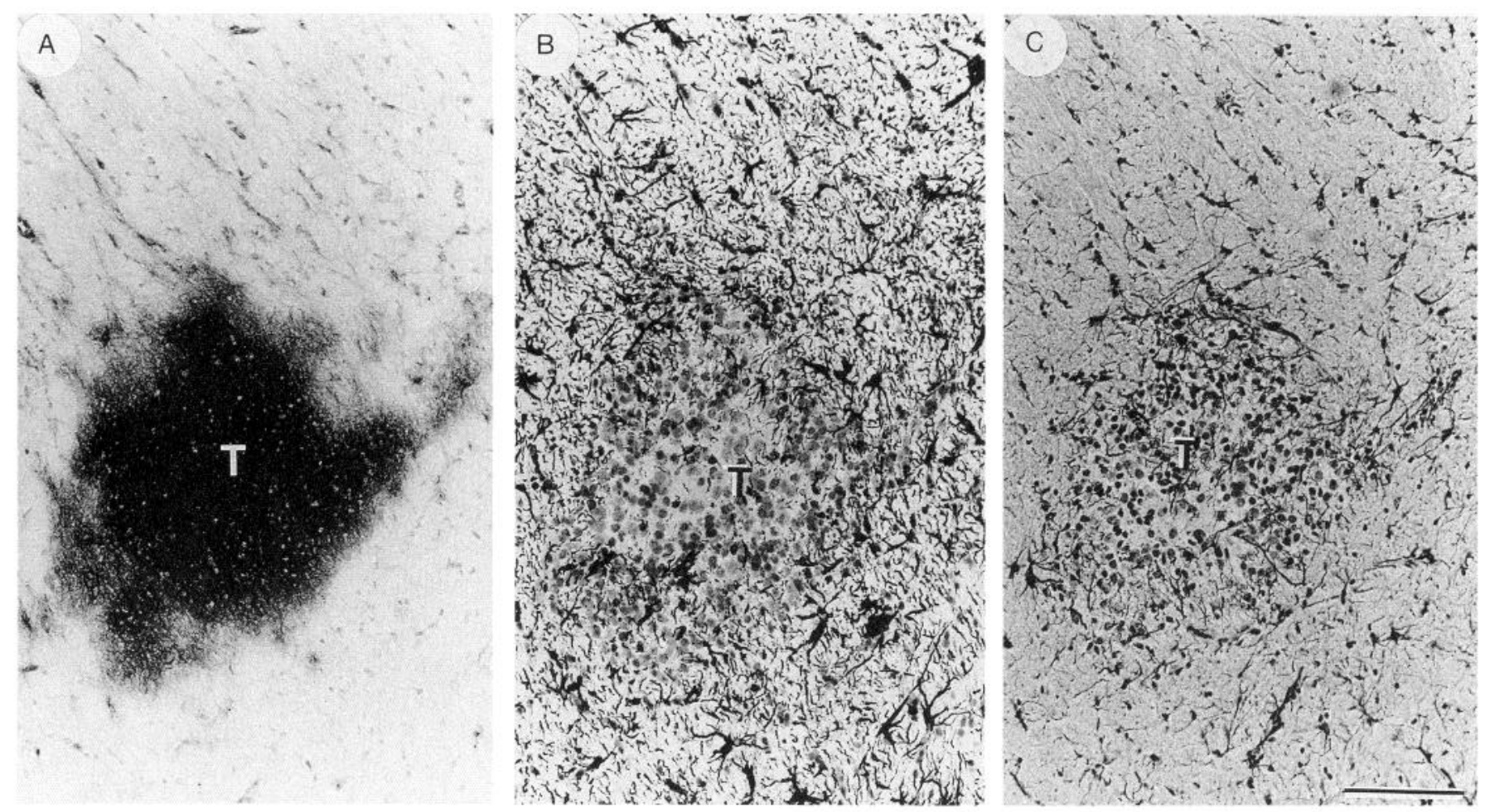

Figure 21. A, A densely M6-positive E14 cortical transplant $(T)$ in the medial edge of the corpus callosum. No long projections are emitted in the plane of section (comparable to the intermediate segment in Fig. $13 C, D$ ). B, Adjacent section showing vimentin-positive astrocytic processes in the surrounding host tract, which are here cut end-on, and vimentin-negative transplant neuropil. $C$, Adjacent section showing the intense GFAP reactivity of the astrocytic processes in the transplant and at the interface, and much weaker GFAP in the host tract; aldehyde fixation. Survival, $6 \mathrm{~d}$. Scale bar, $100 \mu \mathrm{m}$.

matched (olfactory bulb) tissue transplanted into the rostrum of the corpus callosum, and Zwimpfer et al. (1992) have demonstrated the formation of inappropriate synapses by adult retinal ganglion cell axons regenerating along a piece of peripheral nerve led into the cerebellum.

\section{Adult host fiber tracts present a structured environment for donor axon growth}

The structure of adult tracts is built around a longitudinal axis that is defined by the orientation of the axons, the interfascicular rows of glial cell nuclei, and the longitudinal processes of astrocytes (Suzuki and Raisman, 1992; cf. Fig. $1 d$ in Bovolenta et al., 1984). In complex tracts such as the fimbria and corpus callosum, the different fiber contingents interweave as they pass through the tract structure. The microtransplantation technique used in our present studies leaves the internal organization of the host fiber tracts largely undisturbed. Together with the use of small transplants that occupy only restricted parts of the overall host tract, this approach enables us to demonstrate that the routes taken by growing axons are strictly constrained by the local structure of the host fiber tracts.

Where different tracts lie immediately adjacent to each other (such as the fimbria and stria terminalis, or the corpus callosum and cingulum) the glia form a boundary that axons do not cross. The existence of such boundaries in development, and their possible chemical constituents (e.g., Faissner and Kruse, 1990; Snow et al., 1990; Silver et al., 1993) have recently been reviewed (Steindler, 1993). In serial sections of our transplanted material we were able to see that transplants that spanned the boundaries of two tracts gave rise to projections into both tracts (e.g., Fig. 20). However, when the transplants were confined to one side of the boundary, the donor axonal projections remained confined to the tract on that side; that is, despite immediate proximity, donor fibers do not cross intertract boundaries.

\section{Relationship of tract glia to $L I A G$}

The finding that the donor axons grow in parallel to the longitudinal astrocytic processes of the host fiber tracts suggests that the astrocytic processes could be the substrate directing the orientation of the growing donor axons. But although this could be true for the embryo-to-adult transplant situation, it must be borne in mind that at the time in normal brain development when the fimbrial and callosal axons are growing, the configuration of the glia is different, and longitudinal astrocytic processes are absent. Therefore, while LIAG bears some resemblances to normal axon development, it is not a recapitulation, but a new phenomenon.

This can be illustrated by reference to the development of the fimbria (M. Suzuki and G. Raisman, unpublished observations). In the embryonic tract the newly extending axons grow orthogonal to the parallel array of radial glial processes derived from cell bodies along the ventricular surface. During the early postnatal period, when the tract is already well populated with axons, the radial glial tract skeleton is converted to the adult pattern, where astrocytic cell bodies are scattered throughout the width of the tract (cf. Voigt, 1989; Takahashi et al., 1990), and the radial processes are reduced to short, tapering structures that no longer span the full width of the fimbria. The tract astrocytes now generate numerous, untapering longitudinal processes that form an array that clearly delineates the longitudinal axis of the 

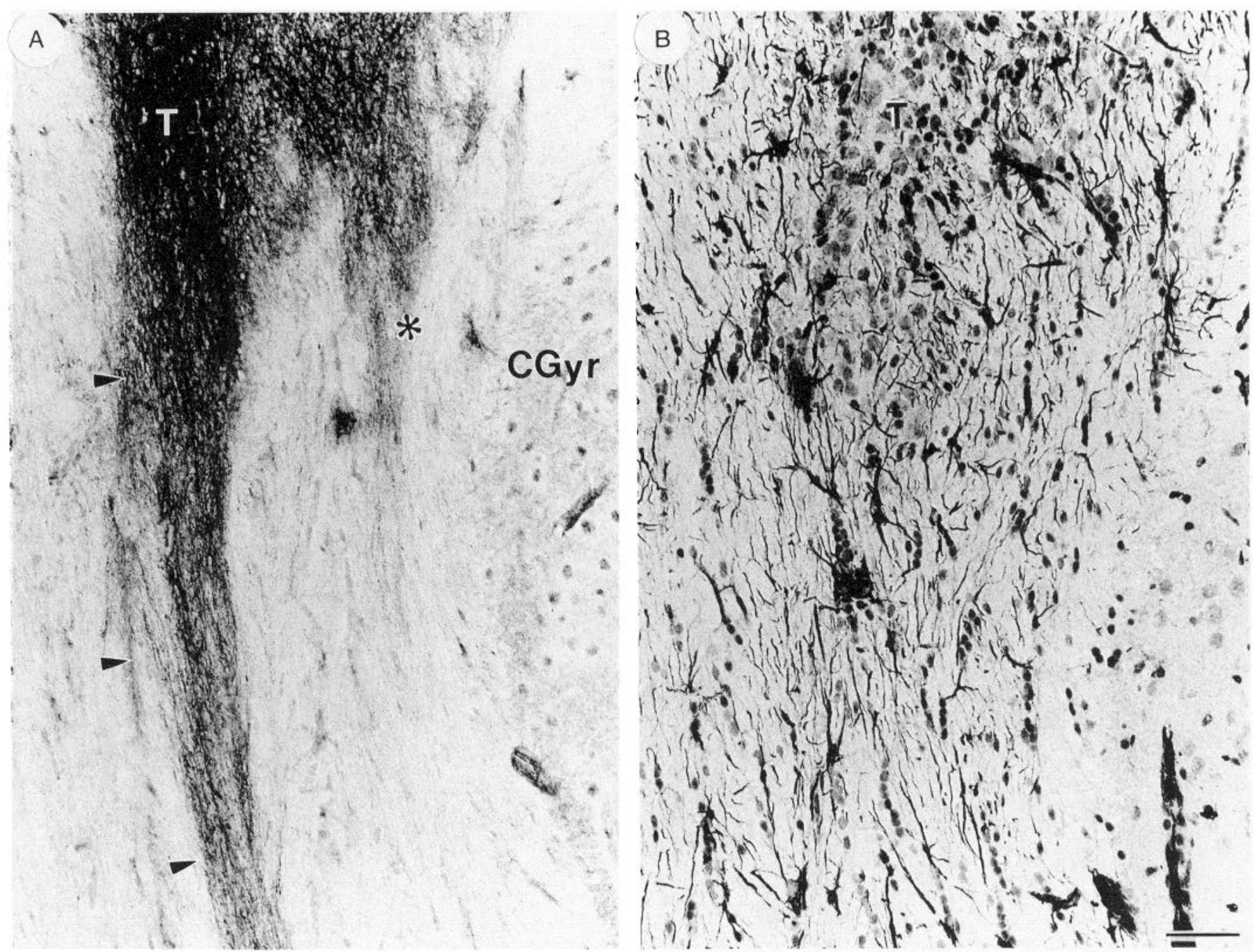

Figure 22. A, Ventral part of a transplant ( $T$ ) of E14 hippocampal cells that lies in the rostrum of the corpus callosum and shows two groups of ventrally directed M6-positive projections (compare Fig. 15A,B), namely, a lateral group (arrowheads) passing ventrally in the corpus callosum, and a medial group (asterisk) passing toward the cortex of the cingulate gyrus (CGyr). B. Adjacent section showing vimentin-positive longitudinal astroglial processes of the corpus callosum are parallel to the trajectory of the axons. Survival, $7 \mathrm{~d}$. Scale bar, $50 \mu \mathrm{m}$.

tract, and runs parallel to the axons and to the rows of postnatally formed oligodendrocytes that later come to flank the astrocytes.

Thus, despite the fact that the adult tract glial skeleton is quite different from that through which the embryonic axons grew, the adult structure still retains cues sufficient to direct rapid and efficient axon growth from embryonic transplants.

\section{Phenotype of intermediate glial filaments (vimentin and GFAP)}

Our analysis of the glial structure of the central tracts was greatly facilitated by the finding that vimentin selectively stained tract (as opposed to neuropil) astrocytes. Conversely, in aldehydefixed material, GFAP favors neuropil astrocytes, which express little vimentin (Bovolenta et al., 1984; Voigt, 1989).

The transplanted tissue itself forms a neuropil (Davies et al., 1993), and here too, in contrast to the adjacent tract astrocytes, the astrocytes are GFAP positive and vimentin negative. Within the transplants, the orientation of the axons (not illustrated) and the GFAP staining of the astrocytic processes show that the internal structure of the transplants is aligned with that of the host tract, suggesting that the development of this transplant neuropil alignment from the initially randomly mixed cell suspension is responding to local orientational cues in the host tract.

There was sufficient overlap of the vimentin and GFAP staining patterns to show that the longitudinal astrocytic processes of the host fiber tract continue uninterrupted through the interface and into the transplant neuropil. These processes would thus be in a position to provide a continuous substrate for the donor axons as they emerge from the transplant and enter the host tract.

During normal development the switch from astrocytic vimentin to GFAP has been correlated with the time of cessation of long axon growth (e.g., Bovolenta et al., 1984). In our transplanted material there are indications that vimentin is upregulated (suggesting a reversion to a more immature phenotype) in the astrocytes of those areas of the host tract through which the newly growing donor axons pass, and intense, sharply localized vimentin expression is induced in the M6-positive column of the otherwise vimentin-negative gray matter of the cingulate cortex. We have not made a systematic investigation of further possible phenotypic changes in the glia associated with 


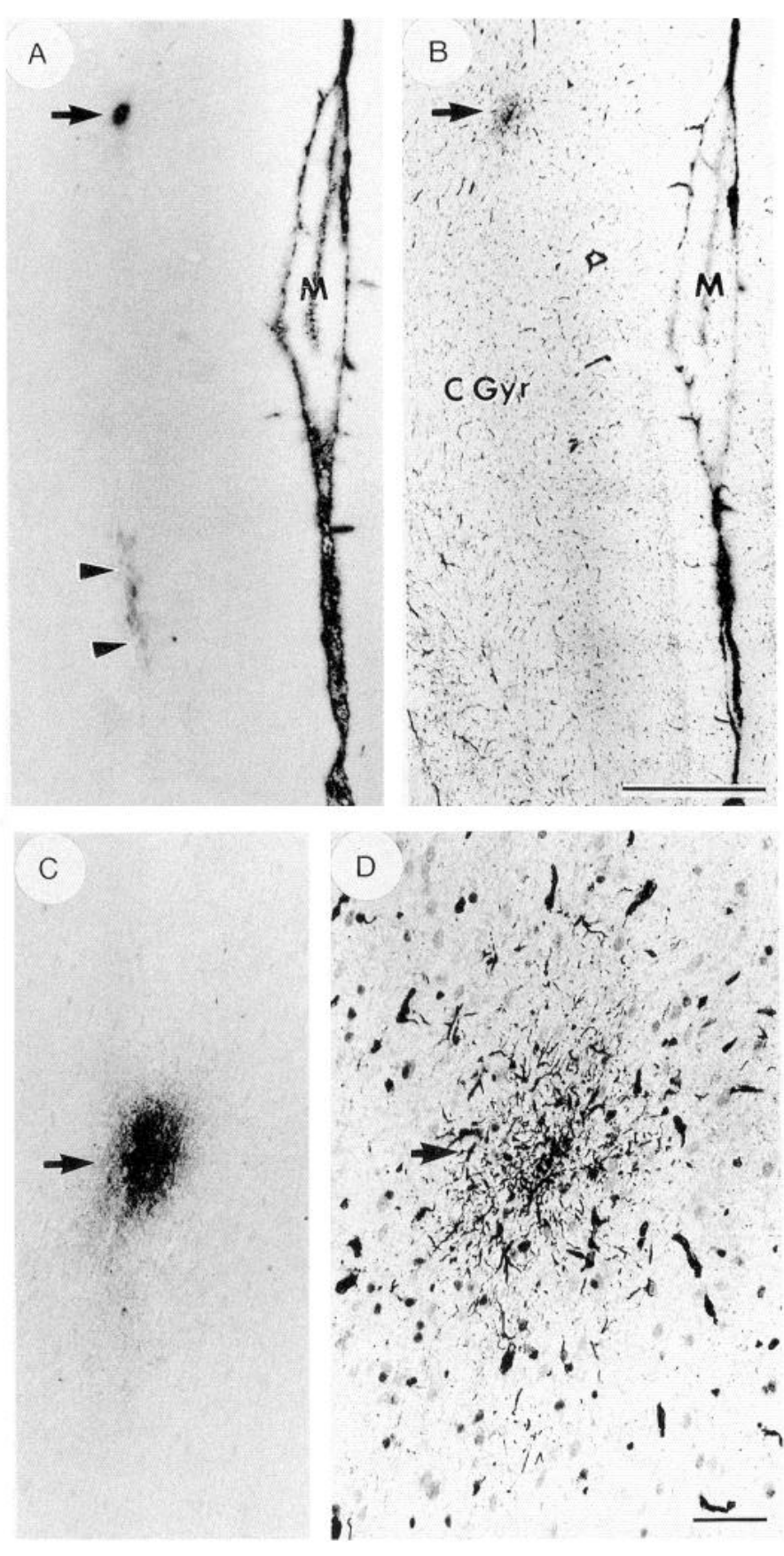

Figure 23. A, Low-power view of a column of M6-positive material (arrow), which arises from a transplant of E14 hippocampal cells placed in the cingulum ventral to the plane of this section, and extends radially for about $1 \mathrm{~mm}$ through the cingulate cortex (CGyr); arrowheads, fascicles of axons in the cingulum; $M$, midline. $B$, Adjacent section stained for vimentin. $C$, Enlarged view of the M6-positive column in $A . D$, Vimentin stain of adjacent section. E14 hippocampal transplant. Survival, $14 \mathrm{~d}$. Scale bars: $A$ and $B, 500 \mu \mathrm{m} ; C$ and $D, 50 \mu \mathrm{m}$.

the newly growing axons (e.g., Schachner, 1991). In the previous study we were unable to detect any upregulation of laminin (cf. Liesi, 1985; Liesi and Silver, 1988).

\section{Possibilities that glia may play a causal role in transplant axon growth}

Is our observed correlation between the orientation of donor axons and that of the host tract astrocytic longitudinal processes

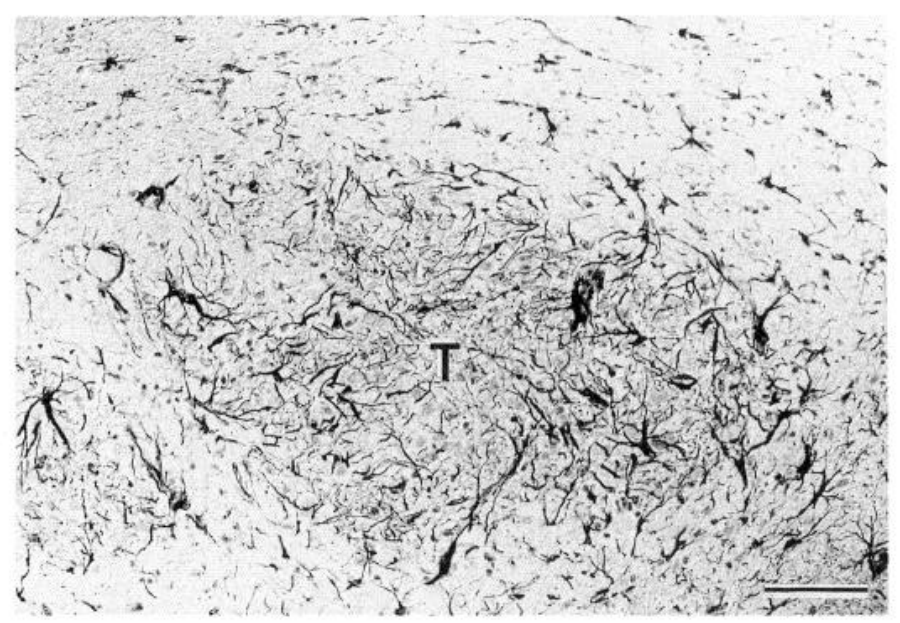

Figure 24. GFAP immunostaining of the neuropil of an E18 cortical transplant $(T)$ in the cingulum to show the swirling pattern of the intratransplant astrocytic processes running to the graft-host interface. At this level the cingulum, which is GFAP poor, is cut almost at right angles to its longitudinal axis. Aldehyde fixation. Survival, $36 \mathrm{~d}$. Scale bar, $100 \mu \mathrm{m}$.

merely circumstantial, or does it indicate that these astroglial processes are necessary for induction or maintenance of LIAG?

There is considerable evidence for a functional effect of glia on neuronal migration and neurite growth. Tissue culture observations indicate that astrocytes play a role in neuronal survival and the formation of neurites (e.g., Lindsay, 1979). Astrocytes from different areas may encode specific information determining the region-specific patterns of axon and dendrite formation (e.g., Rousselet et al., 1988). As shown by the pioneering studies of Rakic (Rakic, 1971, 1972; Nowakowski and Rakic, 1979), developing dentate, neocortical, and cerebellar granule cells migrate to their definitive locations along radial processes (see also Hatten and Mason, 1986; Rickmann et al., 1987), and the intracortical growth of developing callosal axons is similarly linked to radial glia (Norris and Kalil, 1991). In the developing ferret optic nerve, the axon order changes in relation to a specific change from radial to "interfascicular" glia (Guillery and Walsh, 1987), although growth cones do not preferentially associate with glial structures in the developing monkey optic nerve (Williams et al., 1991). However, of the few transplantation studies that have described the host glial arrangement, Silverman et al. (1991) showed that the axons from embryonic transplants did follow host tanycyte processes during their course through the gray matter of the mediobasal hypothalamus to the median eminence in adult hosts.

\section{Relationship of host tract axons to long interfascicular growth of transplant axons}

While a causal correlation of donor axon growth with host glial processes is an attractive hypothesis, we cannot exclude the possibility that the growth and orientation of the donor axons are determined by the host axons, approximately one-third of which are unmyelinated in the fimbria (Wyss et al., 1980; Suzuki and Raisman, 1992). Moreover, degeneration of damaged host axons - and/or the associated glial responses-may also play a part in determining donor axon growth.

Although our method of microtransplantation causes little detectable damage to host fibers or disorganization of the host tract, some host axons would inevitably have been cut (for dis- 
cussion, sec Davies et al., 1993). Preliminary experiments (S. J. A. Davies and G. Raisman, unpublished observations) with complete transection of the host fimbria indicate that the very much higher level of host axon damage thus produced does not appreciably alter the pattern or increase the quantity of LIAG from embryonic donor hippocampal neurons. However, this may indicate that the lower level of host axon damage incurred by microtransplantation alone (i.e., without the added fimbrial section) may have been sufficient to induce the maximal donor axon growth possible.

Degeneration of axons causes responses in tract oligodendrocytes (Ludwin, 1992) and microglia (Schnitzer and Scherer, 1990) as well as astrocytes (Bignami and Dahl, 1976), but our present material does not enable us to say whether glial changes, such as reexpression of astrocytic vimentin (e.g., Takamiya et al., 1988), are caused by degeneration of host axons, or whether they are themselves a response to the growth of the donor axons.

\section{Are the newly formed intrafascicular axons maintained?}

In their studies of fibers in the internal capsule, Wictorin and colleagues showed that in situations where the correct type of donor axons grew through the correct tract, and formed synapses in the appropriately matched denervated host target territory, both PHAL (Wictorin et al., 1990b) and human neurofilament labeling (Wictorin et al., 1990a) demonstrated that axons were still present at survival times after those where we find M6 staining has disappeared. The disappearance of M6 staining in our material (after 7 weeks in the hippocampal and neocortical transplants, and 3 weeks in the E14 superior collicular transplants) could be due to a number of factors associated with axonal maturation, such as downregulation of the molecule that bears the M6 epitope, and/or myelination preventing access of the antibody (Wictorin et al., 1991). It could also be due to retraction of the donor axons, possibly because they did not have access to denervated terminal target structures able to provide the growth factors needed for their survival.

Thus, the LIAG we are observing in these transplantation experiments may represent an early stage of neuronal differentiation, in which "exuberant" axons grow out provisionally, but require to be "validated" by contact with appropriate terminal fields before they are stabilized for permanent existence (O'Leary and Terashima, 1988; O'Leary et al., 1990; Simon and O'Leary, 1992). This could provide a mechanism for adding a subscquent clement of spccificity to the initially nonspecific, bidirectional growth we have observed.

\section{Possible significance of $L I A G$}

The speed, magnitude, and temporal duration of the LIAG which we and others have observed in adult host tracts are comparable to those of axon growth during development in the embryo. The "provisional" nature of LIAG does not necessarily detract from its potential value. On the contrary, it could be an advantage. An inherent ability to eliminate inappropriate axon types would be an asset to any repair strategy.

Although the experimental data so far deal only with embryonic rather than adult axons, the projections formed by transplanted embryonic neurons provide a method for exploring the ability of adult fiber tracts to sustain directed axon growth, and may therefore provide the baseline data needed for future studies of regeneration of cut adult axons. Elucidation of the factors determining this striking LIAG in adult myelinated fiber tracts could be of great importance for future treatments of clinical conditions such as injuries to the internal capsule (stroke) or to the long tracts in the spinal cord (paraplegia).

\section{References}

Barbe MF, Levitt $\mathbf{P}$ (1991) The early commitment of fetal neurons to the limbic cortex. J Ncurosei 11:519-533.

Bignami A, Dahl D (1976) The astroglial response to stabbing. Immunofluorescence studies with antibodies to astrocyte-specific protein (GFA) in mammalian and submammalian vertebrates. Neuropathol Appl Neurobiol 2:99-110.

Bottenstein J, Sato GH (1979) Growth of a rat neuroblastoma cell line in serum-free supplemented medium. Proc Natl Acad Sci USA 76:514-517.

Bovolenta P, Liem RKH, Mason CA (1984) Development of cerebellar astroglia: transitions in form and cytoskeletal content. Dev Biol 102:248-259.

Davies SJA, Field PM, Raisman G (1993) I ong fibre growth by axons of embryonic mouse hippocampal neurons micro-transplanted into the adult rat fimbria. Eur J Neurosci 5:95-106.

Emmett CJ, Jaques-Berg W, Seeley PJ (1990) Microtransplantation of neural cells into adult rat brain. Neuroscience 38:213-222.

Faissner A, Kruse J (1990) Jl/tenascin is a repulsive substrate for central nervous system neurons. Neuron 5:627-637.

Fujii M (1991) Non-specific characteristics of intracerebral elongation from the olfactory bulb transplanted into the young adult host neocortex or hippocampal formation, demonstrated immunohistochemically by the mouse Thy-1 allelic system. Neurosci Res 9:285-291.

Guillery RW, Walsh C (1987) Changing glial organization relates to changing fiber order in the optic nerve of ferrets. J Comp Neurol 265: 203-217.

Hatten MF, Mason C.A (1986) Neuron-astroglia interactions in vitro and in vivo. Trends Neurosci 9:168-174.

Li Y, Raisman G (1994) Long interfascicular axon growth from embryonic mousc hippocampal ncurons transplanted into the myelinated corticospinal tracts and dorsal columns of immunosuppressed adult rat hosts. Brain Res in press.

Liesi P (1985) Laminin-immunoreactive glia distinguish regenerative adult CNS systems from non-regenerative ones. EMBO J 4:25052512 .

Liesi P, Silver J (1988) Is astrocyte laminin involved in axon guidance in the mammalian CNS? Dev Biol 130:774-785.

Lindsay RM (1979) Adult rat brain astrocytes support survival of both NGF-dependent and NGF-insensitive neurons. Nature 282:80-82.

Ludwin SK (1992) Oligodendrocytes from optic nerves subjected to long term Wallerian degeneration retain the capacity to myelinate. Acta Neuropathol (Berl) 84:530-537.

Lund RD, Chang FLF, Hankin MH, Lagenaur CF (1985) Use of a species-specific antibody for demonstrating mousc ncurons transplanted to rat brains. Neurosci Lett 61:221-226.

Nowakowski RS, Rakic P (1979) The mode of migration of neurons to the hippocampus: a Golgi and electron microscopic analysis in fetal rhesus monkey. J Neurocytol 8:697-718.

Norris CR, Kalil K (1991) Guidance of callosal axons by radial glia in the developing cerebral cortex. J Neurosci 11:3481-3492.

O'Leary DDM, Koester SE (1993) Development of projection neuron types, axon pathways, and patterned connections of the mammalian cortex. Neuron 10:991-1006.

O'Leary DDM, Terashima T (1988) Cortical axons branch to multiple subcortical targets by interstitial axon budding: implications for target recognition and "waiting periods." Neuron 1:901-910.

O'Leary DDM, Bicknese AR, De Carlos JA, Heffner CD, Koester SI, Kutka LJ, Terashima T (1990) Target selection by cortical axons: alternative mechanisms to establish axonal connections in the developing brain. Cold Spring Harbor Symp Quant 55:453-468.

Palmgren A (1948) A rapid method for selective silver staining of nerve fibres and nerve endings in mounted paraffin sections. Acta Zool 29:377-392.

Raisman G, Ebner FF (1983) Mossy fibre projections into and out of hippocampal transplants. Neuroscience 9:783-801.

Rakic P (1971) Neuron-glia relationship during granule cell migration in developing cerebellar cortex. A Golgi and electron microscopic study in Macacus rhesus. J Comp Neurol 141:283-312. 
Rakic P (1972) Mode of cell migration to the superficial layers of fetal monkey neocortex. J Comp Neurol 145:61-84.

Ramon y Cajal S (1928) Degeneration and regeneration of the nervous system. New York: Hafner.

Renfranz PJ, Cunningham MG, McKay RDG (1991) Region-specific differentiation of the hippocampal stem cell line HiB5 upon implantation into the developing mammalian brain. Cell 66:713-729.

Reynolds BA, Weiss S (1992) Generation of neurons and astrocytes from isolated cells of the adult mammalian central nervous system. Science 255:1707-1710.

Rickmann M, Amaral DG, Cowan WM (1987) Organization of radial glial cells during the development of the rat dentate gyrus. J Comp Neurol 264:449-479.

Rousselet A, Fetler L, Chamak B, Prochiantz A (1988) Rat mesencephalic neurons in culture exhibit different morphological traits in the presence of media conditioned on mesencephalic or striatal astroglia. Dev Biol 129:495-504.

Schachner M (1991) Cell surface recognition and neuron-glia interactions. Ann NY Acad Sci 633:105-112.

Schnell L, Schwab ME (1990) Axonal regeneration in the rat spinal cord produced by an antibody against myelin-associated neurite growth inhibitors. Nature 343:269-272.

Schnitzer J, Scherer J (1990) Microglial cell responses in the rabbit retina following transection of the optic nerve. J Comp Neurol 302: 779-791.

Schwab ME, Kapfhammer JP, Bandtlow CE (1993) Inhibitors of neurite growth. Annu Rev Neurosci 16:565-595.

Silver J, Edwards MA, Levitt P (1993) Immunocytochemical demonstration of early appearing astroglial structures that form boundaries and pathways along adult tracts in the fetal brain. J Comp Neurol 328:415-436.

Silverman RC, Gibson MJ, Silverman AJ (1991) Relationship of glia to $\mathrm{GnRH}$ axonal outgrowth from third ventricular grafts in hpg hosts. Exp Neurol 1 14:259-274.

Simon DK, O'Leary DDM (1992) Development of topographic order in the mammalian retinocollicular projection. J Neurosci 12:12121232.

Snow DM, Steindler DA, Silver J (1990) Characterization of the glial roof plate of the spinal cord and optic tectum: a possible role for a protcoglycan in the development of an axon barrier. Dev Biol 138: 359-376.

Snyder EY, Deitcher DL, Walsh C, Arnoldaldea S, Hartwieg EA, Cepko CL (1992) Multipotent neural cell lines can engraft and participate in development of mouse cerebellum. Cell 68:33-51.

Sørensen JC, Castro AJ, Klausen B, Zimmer J (1992) Projections from fetal neocortical transplants placed in the frontal neocortex of newborn rats. A Phaseolus vulgaris-leucoagglutinin tracing study. Exp Brain Res 92:299-309.

Sotelo C, Alvarado-Mallart RM (1987) Reconstruction of the defective cerebellar circuitry in adult Purkinje cell degeneration mutant mice hy Purkinje cell replacement through transplantation of solid embryonic implants. Neuroscience 20:1-22

Steindler DA (1993) Glial boundaries in the developing nervous system. Annu Rev Neurosci 16:445 470.

Strömberg I, Bygdeman M, Almqvist P (1992) Target-specific outgrowth from human mesencephalic tissue grafted to cortex or ventricle of immunosuppressed rats. J Comp Neurol 315:445-456.

Suzuki M, Raisman G (1992) The glial framework of central white matter tracts: segmented rows of contiguous interfascicular oligodendrocytes and solitary astrocytes give rise to a continuous meshwork of transverse and longitudinal processes in the adult rat fimbria. Glia $6: 222-235$.
Takahashi T, Misson JP, Caviness VS (1990) Glial process elongation and branching in the developing murine neocortex - a qualitative and quantitative immunohistochemical analysis. J Comp Neurol 302:1528.

Takamiya Y, Kohsaka S, Toya S, Otani M, Tsukada Y (1988) Immunohistochemical studies on the proliferation of reactive astrocytes and the expression of cytoskeletal proteins following brain injury in rats. Dev Brain Res 38:201-210.

Tønder N, Sørensen JC, Bakkum E, Danielsen E, Zimmer J (1988) Hippocampal neurons grafted to newborn rats establish efferent commissural connections. Exp Brain Res 72:577-583.

Tønder N, Sørensen T, Zimmer J (1990) Grafting of fetal C $\wedge 3$ neurons to excitotoxic axon-sparing lesions of the hippocampal CA3 area in adult rats. In: Progress in brain research, Vol 83, Understanding the brain through the hippocampus: the hippocampal region as a model for studying brain structure and function (Storm-Mathisen J, Zimmer J, Ottersen OP, eds), pp 391-409. Amsterdam: Elsevier.

Voigt $T$ (1989) Development of glial cells in the cerebral wall of ferrets: direct tracing of their transformation from radial glia into astrocytes. J Comp Neurol 289:74-88.

Wictorin K, Björklund A (1992) Axon outgrowth from grafts of human embryonic spinal cord in the lesioned adult rat spinal cord. Neuroreport 3:1045-1048.

Wictorin K, Brundin P, Gustavii B, Lindvall O, Björklund A (1990a) Reformation of long axon pathways in adult rat central nervous system by human forebrain neuroblasts. Nature 347:556-558.

Wictorin K, Clarke DJ, Bolam P, Björklund A (1990b) Fetal striatal neurons grafted into the ibotenate lesioned adult striatum: efferent projections and synaptic contacts in the host globus pallidus. Neuroscience 37:301-316.

Wictorin K, Lagenaur CF, Lund RD, Björklund A (1991) Efferent projections to the host brain from intrastriatal striatal mouse-to-rat grafts: time course and tissue-type specificity as revealed by a mouse specific neuronal marker. Eur J Neurosci 3:86-101.

Wictorin K, Brundin P, Sauer H, Lindvall O, Björklund A (1992) Long distance directed axonal growth from human dopaminergic mesencephalic neuroblasts implanted along the nigrostriatal pathway in 6-hydroxydopamine lesioned adult rats. J Comp Neurol 323:475494.

Williams RW, Borodkin M, Rakic P (1991) Growth cone distribution patterns in the optic nerve of fetal monkeys: implications for mechanisms of axon guidance. J Neurosci 11:1081-1094.

Woodhams PL, Webb M, Atkinson DJ, Seeley PJ (1989) A monoclonal antibody, Py, distinguishes different classes of hippocampal neurons. J Neurosci 9:2170-2181.

Wyss JM, Swanson LW, Cowan WM (1980) The organization of the fimbria, dorsal fornix and ventral hippocampal commissure in the rat. Anat Embryol (Berl) 158:303-316.

Zhou CF, Raisman G, Morris RJ (1985) Specific patterns of fibre outgrowth from transplants to host mice hippocampi, shown immunohistochemically by the use of allelic forms of Thy-1. Neuroscience 16:819-833.

Zhou CF, Li Y, Raisman G (1989) Embryonic entorhinal transplants project selectively to the deafferented entorhinal zone of adult mouse hippocampi, as demonstrated by the use of Thy-1 allelic immunohistochemistry. Effect of timing of transplantation in relation to deafferentation. Neuroscience 32:349-362.

Zwimpfer TJ, Aguayo AJ, Bray GM (1992) Synapse formation and preferential distribution in the granule cell layer by regenerating retinal ganglion cell axons guided to the cerebellum of adult hamsters. J Neurosci 12:1144-1159. 